\title{
Bewegungstraining bei Patienten mit chronischen Atemwegserkrankungen: Werden kardiovaskuläre Komorbiditäten und Outcomes berücksichtigt? Eine systematische Übersichtsarbeit
}

\author{
Ana Machado ${ }^{a, b}$ Kirsten Quadflieg ${ }^{a} \quad$ Ana Olveira $^{b-d} \quad$ Charly Keytsman $^{a, e} \quad$ Alda Marques ${ }^{b, f}$ \\ Dominique Hansen ${ }^{\mathrm{a}, \mathrm{e}, \mathrm{g}}$ Chris Burtina, ${ }^{\mathrm{a}}$ \\ ${ }^{a}$ REVAL - Rehabilitation Research Center, Faculty of Rehabilitation Sciences, Hasselt University, Diepenbeek, Belgien; \\ ${ }^{b}$ Respiratory Research and Rehabilitation Laboratory (Lab3R), School of Health Sciences (ESSUA), University of Aveiro, Aveiro, Portugal; \\ 'Respiratory Medicine, West Park Healthcare Centre, Toronto, ON, Kanada; \\ ${ }^{\mathrm{d} S}$ School of Rehabilitation Sciences, Faculty of Health Sciences, McMaster University, Hamilton, ON, Kanada; \\ eBIOMED—Biomedical Research Institute, Hasselt University, Diepenbeek, Belgien; \\ ${ }^{f}$ Institute of Biomedicine (iBiMED), University of Aveiro, Aveiro, Portugal; \\ gJessa Hospital, Heart Centre Hasselt, Hasselt, Belgien
}

\author{
Schlüsselwörter \\ Bewegung · Chronische Lungenerkrankung · \\ Chronisch-obstruktive Lungenerkrankung · COPD · Asthma · \\ Interstitielle Lungenerkrankung · ILD · kardiovaskuläre \\ Komorbiditäten · kardiovaskuläre Outcomes
}

\begin{abstract}
Zusammenfassung
Patienten mit chronisch-obstruktiver Lungenerkrankung (COPD), Asthma und interstitiellen Lungenerkrankungen (ILD) weisen häufig kardiovaskuläre Komorbiditäten (KVK) auf. Bewegungstraining ist eine tragende Säule bei der Behandlung dieser Erkrankungen, doch existieren kaum Empfehlungen zur maßgeschneiderten Anpassung von Programmen für Patienten mit Atemwegserkrankungen und KVK. Ziel der vorliegenden systematischen Übersichtsarbeit war es, die Eignungskriterien zu identifizieren, die für die Auswahl von Patienten mit COPD, Asthma oder ILD und KVK für Bewegungsprogramme herangezogen werden, und die Auswirkungen von Bewegung auf die kardiovaskulären Outcomes zu bewerten. Darüber hinaus sollte festgestellt werden, wie Bewegungsprogramme auf Patienten mit KVK zugeschnitten wurden. Es erfolgte eine Suche in den Datenbanken PubMed, Scopus, Web of Science und Cochrane. Drei Reviewer extrahierten die Daten, und zwei Reviewer bewerteten unabhängig voneinander die Qualität
\end{abstract}

der Studien mithilfe des Quality Assessment Tool for Quantitative Studies. Bei der Berechnung der individuellen und gepoolten Effektstärken (ES) kam MetaXL 5.3 zur Anwendung. In den meisten Studien (58,9\%) waren Patienten mit stabiler und instabiler KVK ausgeschlossen. Insgesamt berichteten 26 von 42 Studien kardiovaskuläre Outcomes. Das am häufigsten berichtete Outcome-Maß war die Herzfrequenz in Ruhe $(\boldsymbol{n}=13)$, und es zeigte sich ein geringer statistisch signifikanter Effekt $(E S=-0,23)$ des Bewegungstrainings auf die Herzfrequenz in Ruhe bei COPD-Patienten. Es wurden keine speziellen Anpassungen für die verordneten Bewegungstrainings beschrieben. Wenige Studien schlossen Patienten mit KVK ein. Ein individueller Zuschnitt der Bewegungsprogramme fehlte und die beobachteten Effekte waren begrenzt. Zukünftige Studien sollten den Effekt maßgeschneiderter Bewegungsprogramme auf relevante Outcome-Maße bei Patienten mit Atemwegserkrankungen und KVK untersuchen.

C일 Die Autoren. Lizenznehmer MDPI, Basel, Schweiz information@karger.com www.karger.com/kkp

Karger ${ }^{\prime \prime}=$
(C) 2020 The Author(s)
Published by S. Karger GmbH, Freiburg Open access

This article is licensed under the Creative Commons Attribution 4.0 International License (CC BY) (http://www.karger.com/Services/Open AccessLicense). Usage, derivative works and distribution are permitted provided that proper credit is given to the author and the original publisher.
Chris Burtin, REVAL

Rehabilitation Research Center

Faculty of Rehabilitation Sciences, Hasselt University

3590 Diepenbeek, Belgien

chris.burtin@uhasselt.be 


\section{Hintergrund}

Chronisch-obstruktive Lungenerkrankung (chronic obstructive pulmonary disease, COPD), Asthma und interstitielle Lungenerkrankungen (interstitial lung diseases, ILD) gehören zu den weltweit am häufigsten auftretenden chronischen Atemwegserkrankungen $[1,2]$. Diese Krankheiten, die mehr als eine Milliarde Menschen betreffen und erhebliche Auswirkungen aufdie Behinderung und Lebensqualität der Patienten (9,5\% der behinderungsbereinigten Lebensjahre im Jahr 2010 [3]) haben, tragen wesentlich zur Krankheitslast bei und zählen zu den häufigsten Todesursachen weltweit (über 3 Millionen Todesfälle im Jahr 2016) [2-4].

Seit einigen Jahren steht der Zusammenhang zwischen chronischen Atemwegserkrankungen und kardiovaskulären Erkrankungen zunehmend im Interesse der klinischen Forschung [5]. Kardiovaskuläre Erkrankungen (wie arterielle Hypertonie, koronare Herzkrankheit, kongestive Herzinsuffizienz, periphere Gefäßerkrankungen und pulmonale Hypertonie) gehören zu den Komorbiditäten mit der höchsten Prävalenz und den stärksten Auswirkungen bei Patienten mit COPD (13\%-68\% der Bevölkerung), Asthma (3\%-25\% der Bevölkerung) und ILD (8\%-86\% der Bevölkerung) [6-13]. Sie verschlechtern den funktionellen Status und die gesundheitsbezogene Lebensqualität der Patienten noch weiter, erhöhen das Hospitalisierungs- und Mortalitätsrisiko (Hazard Ratio (HR) 1,1-3,4 [14-17]) und tragen so zu einer erhöhten wirtschaftlichen und gesellschaftlichen Belastung sowie zu einer Verschlechterung der Prognose bei [7, 9, 10, 12]. Daher sollte bei der Behandlung dieser Patienten der Blick über die Lunge hinaus gehen [18] und eine umfassende Beurteilung sowie Behandlung der kardiovaskulären Komorbiditäten mit maßgeschneiderten Interventionen wurde empfohlen [7, 12, 19, 20].

Bewegungstraining ist eine tragende Säule bei der pulmonalen und kardialen Rehabilitation [21, 22]. Es bewirkt eine Symptomlinderung sowie eine Verbesserung der Funktionsfähigkeit, Belastungstoleranz und gesundheitsbezogenen Lebensqualität von Patienten mit chronischen Atemwegs- und kardiovaskulären Erkrankungen [21,22] und ist daher möglicherweise eine erfolgversprechende Intervention für die Behandlung von Patienten mit diesen Begleiterkrankungen. Studien haben allerdings gezeigt, dass die Effekte bei Patienten mit chronischen Atemwegserkrankungen und gleichzeitig vorliegenden kardiovaskulären Komorbiditäten in der Regel geringer ausfallen als bei Patienten ohne kardiovaskuläre Komorbiditäten [7, 19, 23]. Zudem existieren kaum Empfehlungen dazu, wie Bewegungsprogramme in Hinblick auf kardiovaskuläre Begleiterkrankungen bei COPD, Asthma und ILD angepasst werden können.

Zur Untermauerung evidenzbasierter Aussagen zielte die vorliegende systematische Übersichtsarbeit deshalb darauf ab: 1. zu ermitteln, welche Eignungskriterien in Bezug auf kardiovaskuläre Erkrankungen herangezogen wurden, um Patienten mit COPD, Asthma oder ILD für Studien auszuwählen, in denen die Wirksamkeit von Bewegungsprogrammen über eine Dauer von mindestens drei Monaten untersucht wurde, 2. die Auswirkungen eines mindestens dreimonatigen Bewegungstrainings auf die kar- diovaskulären Outcomes dieser Patienten zu bewerten und 3. festzustellen, wie die Bewegungsprogramme auf die kardiovaskulären Komorbiditäten der Patienten zugeschnitten wurden.

\section{Methoden}

\section{Suchstrategie}

Die vorliegende systematische Übersichtsarbeit folgte bei der Berichterstattung den Preferred Reporting Items for Systematic reviews and Meta-Analyses (PRISMA)-Richtlinien [24] und wurde in zwei Phasen durchgeführt. In Phase 1 wurde ermittelt, welche Eignungskriterien zur Auswahl von Patienten mit kardiovaskulären Komorbiditäten in klinischen Studien zur Wirksamkeit von Bewegungsprogrammen herangezogen wurden. In Phase 2 wurde der Einfluss des Bewegungstrainings auf die kardiovaskulären Outcomes bewertet und untersucht, wie die Bewegungsprogramme auf Patienten mit kardiovaskulären Komorbiditäten zugeschnitten wurden.

Im Mai 2019 erfolgte eine systematische Literaturrecherche in den folgenden elektronischen Datenbanken: PubMed, Scopus, Web of Science und Cochrane. Die Suchbegriffe waren auf Titel, Abstracts und Schlüsselwörter/MeSH-Termini beschränkt. Die vollständige Suchstrategie ist in Appendix A (Original abrufbar unter www. mdpi.com/2077-0383/8/9/1458/htm\#app1-jcm-08-01458) beschrieben.

\section{Eignungskriterien und Auswahl der Studien}

In Phase 1 wurden Studien eingeschlossen, die 1. erwachsene Patienten mit stabiler COPD, Asthma und/oder ILD (d.h. seit 4 Wochen keine Exazerbation) untersuchten, 2. als Intervention ein mindestens 12-wöchiges Bewegungstraining (d.h. Ausdauerund/oder Krafttraining) beinhalteten [25], 3. mindestens zwei unter direkter Aufsicht durchgeführte Trainingseinheiten pro Woche vorsahen [26], und bei denen es sich 4 . um prospektive quantitative Originalstudien handelte, die 5. in portugiesischer, englischer, französischer, niederländischer oder spanischer Sprache verfasst waren. Retrospektive Studien, Fallstudien, Fallserien, Abstracts und Studien, die andere Bewegungsmodalitäten beinhalteten (z.B. Yoga, Tai-Chi oder Qigong) wurden ausgeschlossen. Nach Bereinigung von Dubletten nahmen drei Reviewer (A.M., K.Q. und A.O.) eine Bewertung aller identifizierten, potenziell infrage kommenden Studien vor. Die Auswahl der Studien erfolgte auf Grundlage von Studientiteln und Abstracts. Waren Titel und Abstract für den Zweck der Übersichtsarbeit potenziell relevant, dann lasen die Reviewer den Volltext sorgfältig durch und entschieden über den Einschluss der Studie. Bei etwaigen Differenzen wurde ein vierter Reviewer (C.B.) hinzugezogen.

In Phase 2 wurden Studien aufgenommen, die in Phase 1 eingeschlossen worden waren und Angaben zur Prävalenz kardiovaskulärer Komorbiditäten (d.h. alle kardiovaskulären Erkrankungen, die zusätzlich zur Atemwegserkrankung vorlagen und durch eine objektive Untersuchung der Patienten, Überprüfung der Patientenakte oder Selbstauskunft der Patienten bezüglich ihrer Begleiterkrankungen festgestellt wurden) in den Baseline-Merkma- 
len der untersuchten Patientenpopulation enthielten und/oder in denen mindestens ein kardiovaskuläres Outcome (d.h. Herzfrequenz, systolischer und diastolischer Blutdruck, flussvermittelte Vasodilatation, Pulswellengeschwindigkeit, Intimadicke der Arteria carotis, Herzfunktion und -struktur, Herzfrequenzvariabilität, EKG-Analyse und Serumlipidprofil) angegeben war.

\section{Qualitätsbeurteilung und Datenextraktion}

Zwei Reviewer (K.Q. und A.O.) beurteilten unabhängig voneinander die Qualität der in Phase 2 aufgenommenen Studien mithilfe des Quality Assessment Tool for Quantitative Studies, das vom Effective Public Health Practice Project, Kanada, entwickelt worden war [27]. Dieses Instrument dient der Bewertung von sechs Domänen der methodischen Qualität: 1. Selektionsbias, 2. Studiendesign, 3. Confounders, 4. Verblindung, 5. Datenerhebungsmethoden und 6. Withdrawals und Dropouts [27]. Dabei wird jede Domäne gemäß einem standardisierten Leitfaden als «stark», «mäßig» oder «schwach» eingestuft, und die Gesamtbewertung der Studie erfolgt auf Grundlage der Gesamtzahl an «starken» und «schwachen» Einstufungen [27].

In Phase 1 wurden aus allen eingeschlossenen Studien die Daten zu den Auswahlkriterien (d.h. Einschluss- und Ausschlusskriterien), die zur Auswahl der Patienten für die Studie verwendet worden waren, extrahiert. Danach wurden alle Erkrankungen, die ein etwaiger Hinderungsgrund für die Teilnahme der Patienten an den Bewegungsprogrammen gewesen wären, und die als Gründe für den Einschluss (z.B. Fehlen einer schweren kardiovaskulären Erkrankung) oder den Ausschluss (z.B. Vorliegen einer schweren kardiovaskulären Erkrankung) dieser Patienten angegeben wurden, zusammengetragen und als Ausschlusskriterien berichtet. Darüber hinaus erfolgte eine Datenextraktion aus den in Phase 2 eingeschlossenen Studien in ein zuvor entwickeltes strukturiertes Tabellenformat, das folgende Themen umfasste: Studie (Erstautor, Jahr der Veröffentlichung, Land), Studiendesign, Patientenpopulation (Teilnehmerzahl, Diagnose, Alter, Geschlecht, forciertes exspiratorisches Volumen in $1 \mathrm{~s}$ (forced expiratory volume in second 1, $\mathrm{FEV}_{1}$ ), forcierte Vitalkapazität (forced vital capacity, FVC), Kohlenmonoxid-Diffusionskapazität (diffusing capacity for carbon monoxide, DLCO), Intervention (Art und Intensität der Intervention), Dauer und Häufigkeit (Dauer der Intervention, Dauer und Häufigkeit der Trainingseinheiten), Outcome und Outcome-Maß sowie Ergebnisse. Im Rahmen dieser Übersichtsarbeit wurden nur kardiovaskuläre Outcomes und Outcome-Maße berücksichtigt.

\section{Datenanalyse und -synthese}

Um die Konsistenz der Qualitätsbeurteilung beider Reviewer zu untersuchen, wurde eine Analyse der Inter-Rater-Übereinstimmung mittels Cohen-Kappa-Koeffizient durchgeführt. Der Wert des Cohen-Kappa-Koeffizienten liegt zwischen 0 und 1 und kann als «schwache» $(\leq 0,2)$, «leichte» $(0,21-0,4)$, «moderate» $(0,41-$ $0,6)$, «hohe» $(0,61-0,8)$ oder «(fast) vollständige» $(\geq 0,81)$ Übereinstimmung interpretiert werden [28]. Die statistische Analyse erfolgte mittels IBM SPSS 24.0 (IBM, Armonk, New York, NY, USA).

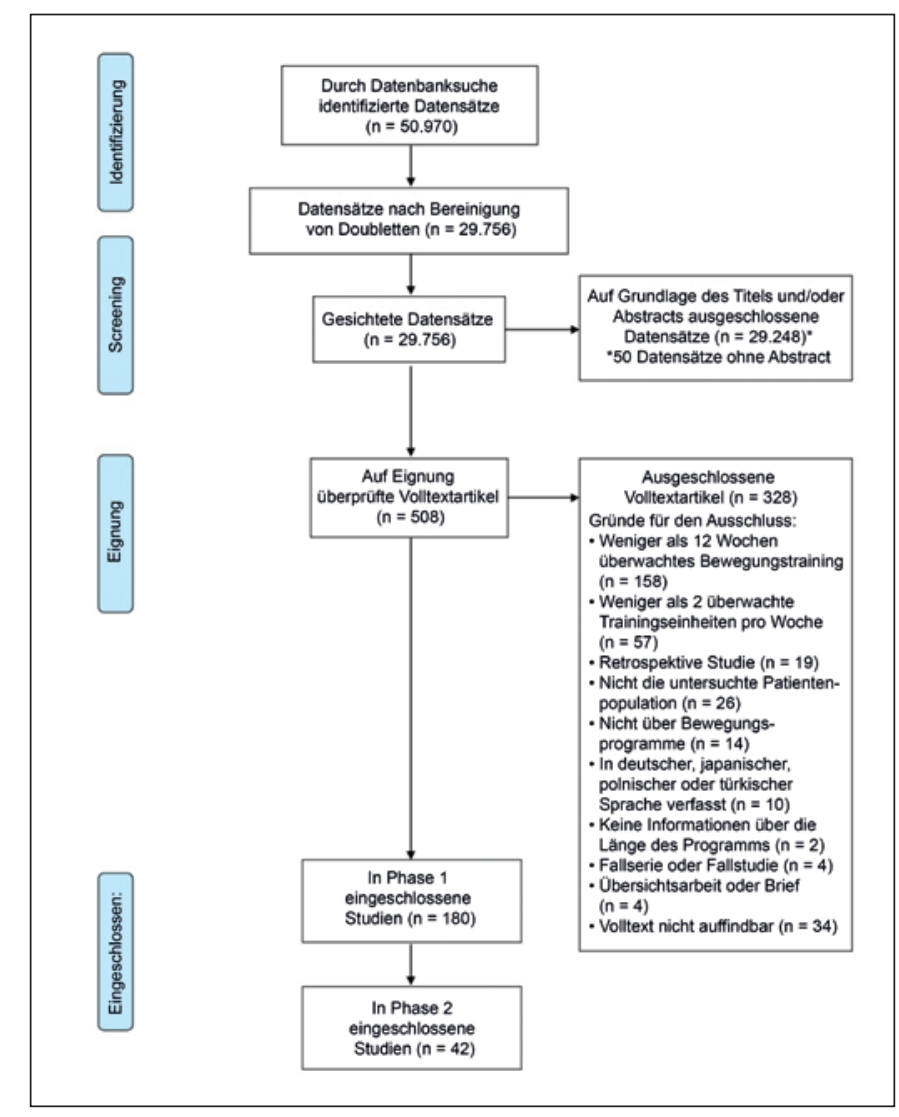

Abb. 1. PRISMA-Flowchart der eingeschlossenen Studien.

Wenn möglich, wurden die Effektstärken (ES) berechnet und es erfolgte eine Metaanalyse. Die Effektstärken wurden als Cohen'sd-Wert auf Grundlage der Prä/Post-Mittelwerte und Standardabweichungen oder Mittelwertdifferenzen und Standardabweichungen nach der Formel von Morris berechnet [29], und als «klein» $(\geq 0,2)$, «mittelmäßig» $(\geq 0,5)$ oder «groß» $(\geq 0,8)$ interpretiert [30]. Für die Metaanalyse wurde MetaXL 5.3 verwendet. Die Berechnung der gepoolten Effektschätzungen erfolgte mithilfe der Inverse-Varianz-Methode unter Annahme eines Modells mit festen Effekten. Input-Daten waren der Cohen's d-Wert jeder Studie und der jeweilige Standardfehler. Output-Daten waren der gepoolte Cohen's-d-Wert und die entsprechenden Konfidenzintervalle. Zur Beurteilung der Homogenität der Studien wurden der Cochran's-Q-Test und die I²-Statistik verwendet.

\section{Ergebnisse}

\section{Auswahl der Studien}

Die Literatursuche ergab eine Gesamtzahl von 50.970 Datensätzen. Nach Bereinigung der Dubletten wurden 29.756 Datensätze anhand von Titel und Abstract auf relevante Inhalte gesichtet und 29.248 Datensätze wurden ausgeschlossen. Von 508 potenziell relevanten Artikeln wurde die Volltextversion beurteilt und 180 dieser Artikel wurden in Phase 1 und 42 in Phase 2 eingeschlossen (Abb. 1).
180

Kompass Pneumol 2020;8:178-199 DOI: $10.1159 / 000509305$ 


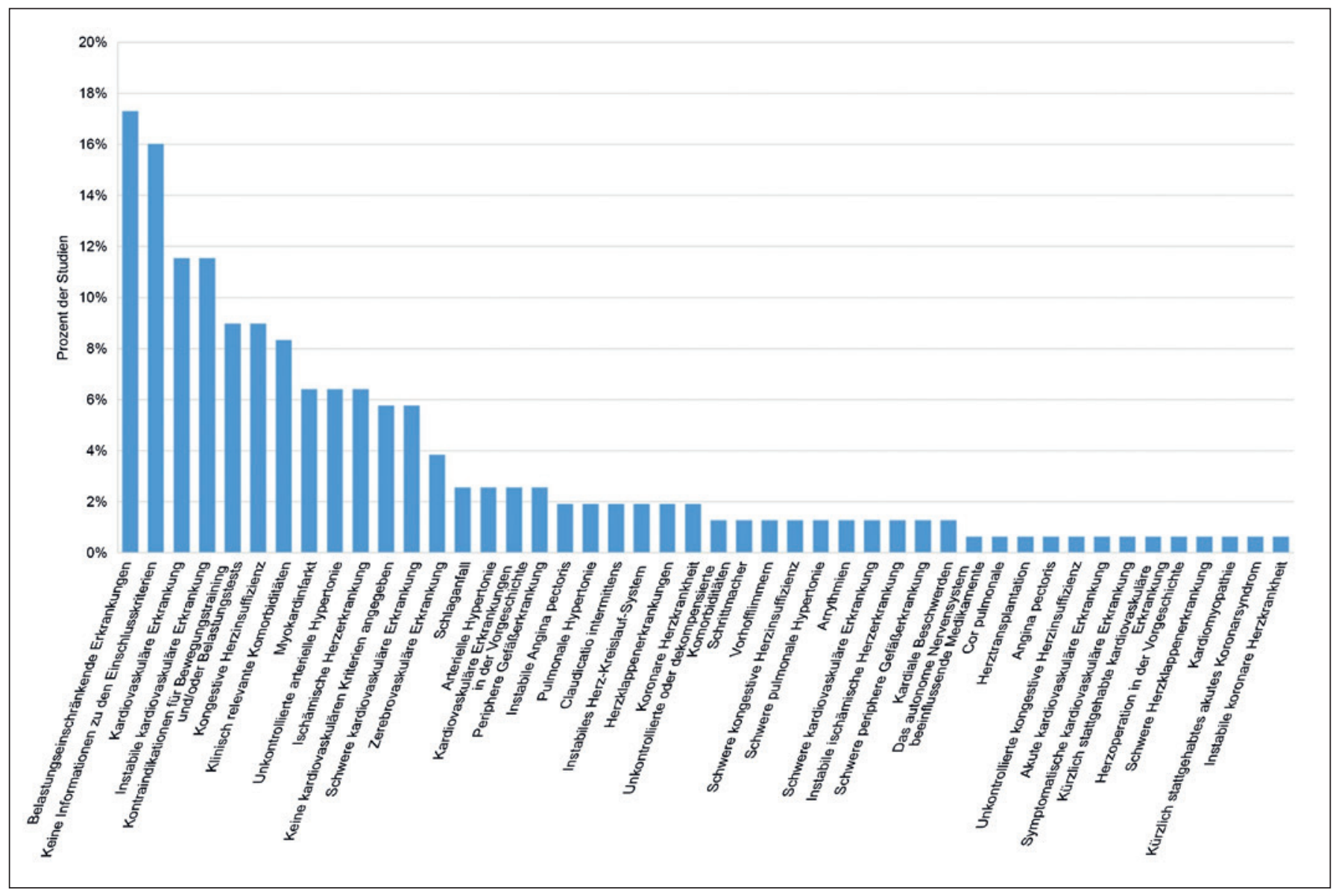

Abb. 2. Ausschlusskriterien von Studien mit COPD-Patienten ( $n=156$ Studien).

Phase 1: Kriterien zum Ausschluss von Patienten mit kardiovaskulären Komorbiditäten von Bewegungsprogrammen Die 180 eingeschlossenen Studien waren zwischen 1987 und 2019 durchgeführt worden. Insgesamt schlossen 156 Studien Patienten mit COPD ein [23, 31-185], 15 Studien schlossen Patienten mit Asthma ein [46, 52, 63, 78, 185-195] und 16 Studien schlossen Patienten mit ILD ein [87, 185, 196-209].

In den Studien mit COPD-Patienten fanden sich 44 verschiedene Ausschlusskriterien (Abb. 2). Von diesen waren belastungseinschränkende Erkrankungen [34, 36, 40, 53, 60, 61, 89, 92, 104, 114, $125,134,135,138,148,157,166-170,173,174,179,181,183,184]$ $(\mathrm{n}=27 ; 17,3 \%)$ das am häufigsten berichtete Ausschlusskriterium, gefolgt von allgemeiner kardiovaskulärer Erkrankung [32, 39, 44, $70,74,75,94,100,103,107,109,112,130,152,153,160,164,171]$ ( $\mathrm{n}=18 ; 11,5 \%)$ und instabiler kardiovaskulärer Erkrankung 38, $47,53,65,66,98,99,101,108,116-119,133,137,146,147,180]$ (n $=18 ; 11,5 \%)$. Vierunddreißig Studien $(21,8 \%)[23,48,50,55,63$, $67,69,73,77-80,83,84,86,88,91,95,97,110,111,115,122,126$, $127,132,142,149,150,155,161,165,175,185]$ enthielten keine Angaben zu den Eignungskriterien für kardiovaskuläre Komorbiditäten.

In Studien mit Asthma-Patienten fanden sich 10 verschiedene Ausschlusskriterien (Abb. 3). Allgemeine kardiovaskuläre Er- krankung $[186,191,192](\mathrm{n}=3 ; 20,0 \%)$ war das am häufigsten berichtete Kriterium, gefolgt von Kontraindikationen für Bewegungstraining und/oder Belastungstests $[194,195](n=2 ; 13,3 \%)$. Sieben Studien $(46,7 \%)$ [63, 78, 185, 187, 189, 190, 193] machten keine Angaben zu den Ausschlusskriterien.

In Studien mit Patienten mit ILD waren 10 verschiedene Ausschlusskriterien angegeben (Abb. 4). Von diesen waren instabile kardiovaskuläre Erkrankungen [198, 199, 202, 203, 208, 209] ( $\mathrm{n}=$ 6;37,5\%) das am häufigsten berichtete Kriterium gefolgt von Kontraindikationen für Bewegungstraining und/oder Belastungstests (z.B. instabile Angina, kürzlich stattgehabter Myokardinfarkt oder zerebrovaskuläres Ereignis) [201, 204, 207] ( $\mathrm{n}=3$; 18,8\%). Vier Studien $(25,0 \%)[185,197,205,206]$ enthielten keine Angaben zu Ausschlusskriterien.

Allgemein enthielten $22,8 \%$ der Studien $[23,48,50,55,63,67,69$, $73,77-80,83,84,86,88,91,95,97,110,111,115,122,126,127,132$, $142,149,150,155,161,165,175,185,187,189,190,193,197,205$, 206] keine Informationen über die Eignungskriterien, $18,3 \%$ der Studien $[33,42,54,65,66,72,82,96,98,99,101,108,116-119,128$, $137,141,143,145,146,180,182,194,195,200-203,207-209]$ schlossen nur Patienten mit akuten oder instabilen kardiovaskulären Komorbiditäten, die eine Kontraindikation für Bewegungstraining darstellten, aus, und 58,9\% der Studien $[31,32,34-41$, 


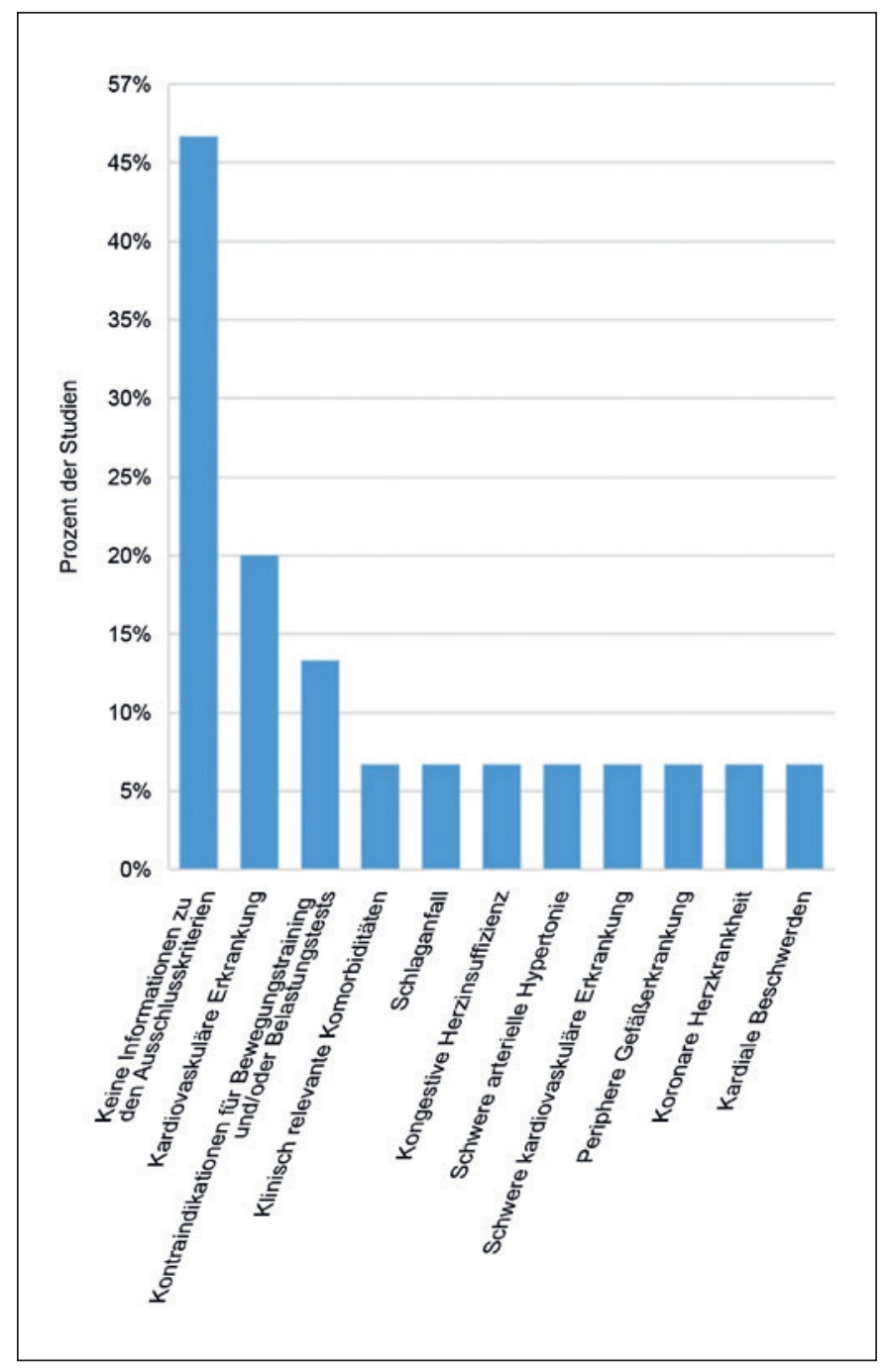

Abb. 3. Ausschlusskriterien von Studien mit Asthma-Patienten ( $\mathrm{n}=15$ Studien).

$43-47,49,51-53,56-62,64,68,70,71,74-76,81,85,87,89,90$, $92-94,100,102-107,109,112-114,120,121,123-125,129-131$, $133-136,138-140,144,147,148,151-160,162-164,166-174,176-$ 179, 181, 183, 184, 186, 188, 191, 192, 196, 198, 199, 204] schlossen sowohl stabile als auch instabile kardiovaskuläre Komorbiditäten aus.

Phase 2: Auswirkungen des Bewegungstrainings auf die kardiovaskulären Outcomes und Design der

Bewegungsprogramme Qualitätsbeurteilung

Die Ergebnisse der Beurteilung der methodischen Qualität sind in Tabelle 1 dargestellt. Die meisten Studien $(\mathrm{n}=24 ; 57,1 \%)$ waren von schwacher Qualität, es zeigte sich eine hohe Übereinstimmung zwischen den beiden Reviewern ( $\mathrm{k}=0,72$; 95\%-Konfidenzintervall $(\mathrm{KI})=0,53-0,91 ; \mathrm{p}<0,001$; Prozent Übereinstimmung $=85,7 \%$ ).

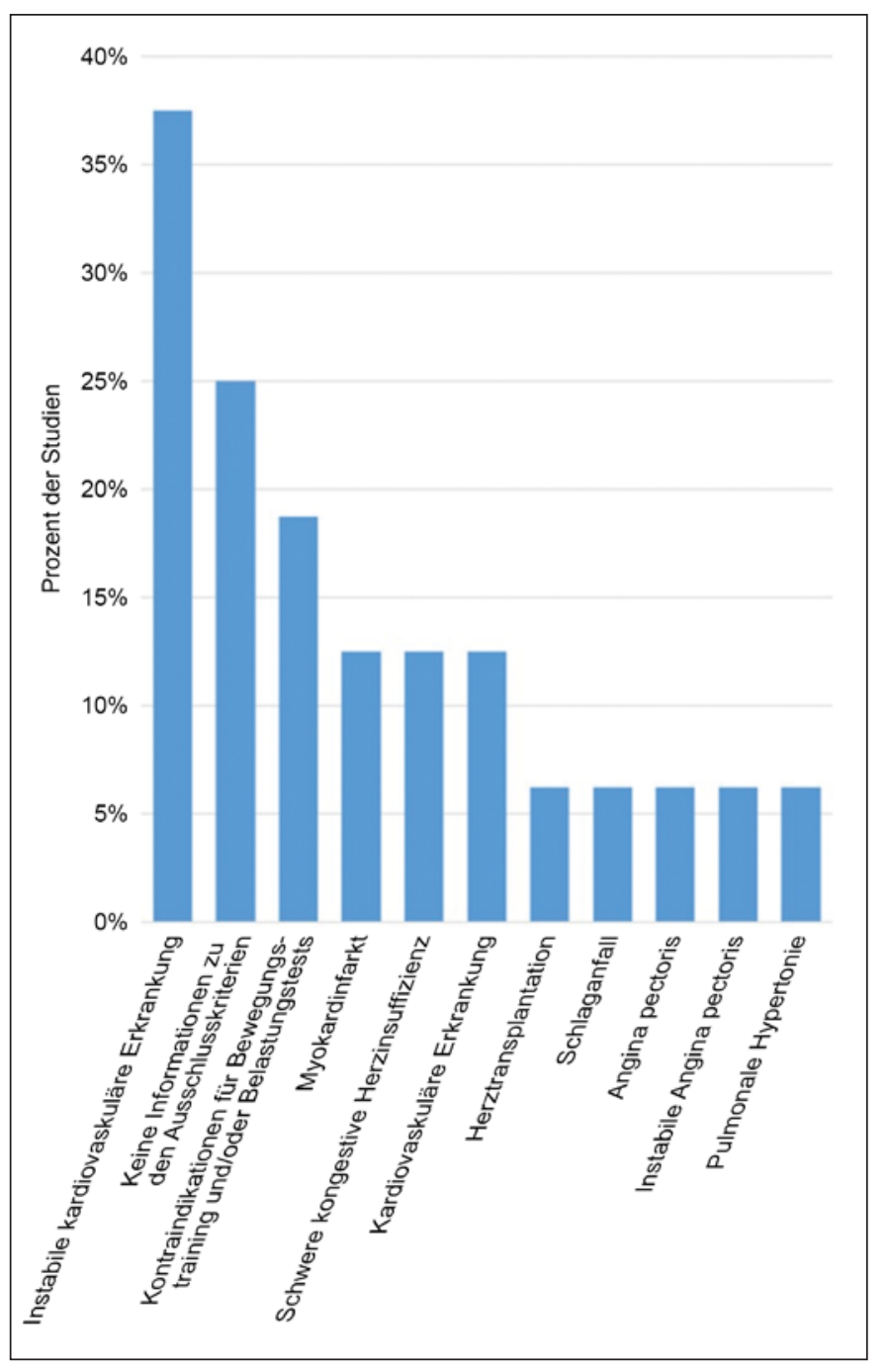

Abb. 4. Ausschlusskriterien von Studien mit Patienten mit interstitiellen Lungenerkrankungen (ILD) ( $\mathrm{n}=16$ Studien).

\section{Studiencharakteristika}

Die Charakteristika der eingeschlossenen Studien sind in Tabelle 2, Tabelle 3 und Tabelle 4 dargestellt. Von den 42 eingeschlossenen Studien schlossen 32 Patienten mit COPD ein [33, 37, 42-44, $48,49,53-55,57,59,68,80,85,90,101,104,106-108,120,121,123$, $124,128,129,136,146,147,151,167], 2$ Studien schlossen Patienten mit Asthma ein $[186,189]$ und 8 Studien schlossen Patienten mit ILD ein [196, 198, 201-203, 207-209]. Bei den meisten Studien handelte es sich um randomisierte kontrollierte Studien ( $\mathrm{n}=24$; $57,1 \%)[37,43,44,49,53,80,85,120,121,123,124,129,146,147$, $151,167,186,189,196,198,202,207-209]$.

Insgesamt wurden 1704 Patienten (65,2\% Männer; Daten aus 34 Studien) mit einem gewichteten Durchschnittsalter von 65,4 Jahren und einem mittleren $\mathrm{FEV}_{1}$ von 53,7\% des Sollwerts (Daten aus 36 Studien) in die eingeschlossenen Studien aufgenommen.

Nur 13 Studien mit COPD-Patienten [33, 42-44, 48, 54, 55, 59, 68, $85,101,151,167]$ und 6 Studien mit ILD-Patienten [196, 198, 203, 207-209] enthielten in den Baseline-Merkmalen der Patientenpo- 
Tab. 1. Qualitätsbewertung der in Phase 2 eingeschlossenen Studien $(n=42)$ mithilfe des Quality Assessment Tool for Quantitative Studies

\begin{tabular}{|c|c|c|c|c|c|c|c|}
\hline Studie & Selektionsbias & Studiendesign & Confounder & Verblindung & $\begin{array}{l}\text { Datenerhebungs- } \\
\text { methode }\end{array}$ & $\begin{array}{l}\text { Withdrawals und } \\
\text { Drop-outs }\end{array}$ & $\begin{array}{l}\text { Global } \\
\text { Rating }\end{array}$ \\
\hline Cochrane et al., 1990 & 3 & 1 & 1 & 3 & 1 & 3 & 3 \\
\hline Berry et al., 1999 & 2 & 2 & 3 & 3 & 1 & 1 & 3 \\
\hline Foy et al., 2001 & 3 & 1 & 3 & 3 & 1 & 1 & 3 \\
\hline Berry et al., 2003 & 2 & 1 & 1 & 2 & 1 & 1 & 1 \\
\hline Panton et al., 2004 & 2 & 1 & 3 & 3 & 1 & 1 & 3 \\
\hline Marquis et al., 2008 & 2 & 1 & 3 & 1 & 1 & 3 & 3 \\
\hline Averna et al., 2009 & 3 & 1 & 1 & 3 & 1 & 1 & 2 \\
\hline Berry et al., 2010 & 3 & 1 & 1 & 2 & 1 & 1 & 2 \\
\hline Rejbi et al., 2010 & 2 & 1 & 2 & 3 & 1 & 1 & 2 \\
\hline Camillo et al., 2011 & 2 & 1 & 1 & 3 & 1 & 1 & 2 \\
\hline Lan et al., 2011 & 3 & 2 & 2 & 3 & 1 & 3 & 3 \\
\hline Corhay et al., 2012 & 3 & 2 & 3 & 2 & 1 & 2 & 3 \\
\hline Georgiopoulou et al., 2012 & 2 & 2 & n.z. & 3 & 1 & 1 & 2 \\
\hline Lan et al., 2013 & 2 & 2 & n.z. & 3 & 1 & 3 & 3 \\
\hline Cheng et al., 2014 & 3 & 2 & n.z. & 3 & 1 & 3 & 3 \\
\hline Gaunaurd et al., 2014 & 3 & 1 & 1 & 3 & 1 & 1 & 3 \\
\hline Vainshelboim et al., 2014 & 3 & 1 & 1 & 3 & 1 & 1 & 3 \\
\hline Borghi-Silva et al., 2015 & 2 & 1 & 1 & 2 & 1 & 1 & 1 \\
\hline Campos et al., 2015 & 2 & 2 & n.z. & 3 & 1 & 1 & 2 \\
\hline Leite et al., 2015 & 2 & 1 & 1 & 3 & 1 & 3 & 3 \\
\hline Marcellis et al., 2015 & 2 & 2 & n.z. & 3 & 1 & 2 & 2 \\
\hline Mkacher et al., 2015 & 2 & 1 & 1 & 3 & 1 & 1 & 2 \\
\hline Spielmanns et al., 2015 & 3 & 1 & 1 & 2 & 1 & 3 & 3 \\
\hline Vainshelboim et al., 2015 & 2 & 1 & 1 & 3 & 1 & 1 & 2 \\
\hline Boström et al., 2016 & 3 & 1 & 1 & 2 & 1 & 1 & 2 \\
\hline Cardoso et al., 2016 & 2 & 1 & 3 & 3 & 1 & 1 & 3 \\
\hline El-Kader et al., 2016 & 3 & 1 & 1 & 3 & 1 & 1 & 3 \\
\hline Engel et al., 2016 & 3 & 1 & 1 & 1 & 1 & 1 & 2 \\
\hline Boeselt et al., 2017 & 3 & 1 & 1 & 3 & 1 & 2 & 3 \\
\hline Kanao et al., 2017 & 3 & 2 & n.z. & 3 & 1 & 1 & 3 \\
\hline Pacheco et al., 2017 & 2 & 3 & n.z. & 3 & 1 & 1 & 3 \\
\hline Papp et al., 2017 & 3 & 1 & 3 & 3 & 1 & 2 & 3 \\
\hline Vainshelboim et al., 2017 & 2 & 1 & 1 & 3 & 1 & 1 & 2 \\
\hline Vasilopoulou et al., 2017 & 3 & 1 & 3 & 3 & 1 & 1 & 3 \\
\hline Lan et al., 2018 & 3 & 2 & n.z. & 3 & 1 & 1 & 3 \\
\hline Moezy et al., 2018 & 3 & 1 & 1 & 3 & 1 & 1 & 3 \\
\hline Naz et al., 2018a & 2 & 2 & n.z. & 3 & 1 & 1 & 2 \\
\hline Naz et al., 2018b & 3 & 1 & 1 & 3 & 1 & 1 & 3 \\
\hline Silva et al., 2018 & 2 & 1 & 1 & 3 & 1 & 1 & 2 \\
\hline Charikiopoulou et al., 2019 & 2 & 2 & 3 & 3 & 1 & 1 & 3 \\
\hline Mekki et al., 2019 & 2 & 1 & 1 & 2 & 1 & 2 & 2 \\
\hline Silva et al., 2019 & 2 & 1 & 1 & 3 & 1 & 1 & 2 \\
\hline
\end{tabular}

Legende: 1 = hohe Qualität; 2 = mäßige Qualität; 3 = schwache Qualität; n.z:: nicht zutreffend.

pulation Angaben zu Patienten mit kardiovaskulären Komorbiditäten. Es wurden keine Studien gefunden, in denen die Aufnahme von Asthma-Patienten mit kardiovaskulären Komorbiditäten berichtet wurde. Studien mit COPD-Patienten schlossen Patienten mit arterieller Hypertonie [33, 42-44, 48, 54, 68, 85, 151] (9 Studien; 314 Patienten), kardiovaskulären Erkrankungen [43, 44, 48, 55, 59, 85, 101, 151, 167] (9 Studien; 247 Patienten), Durchblutungsstörungen [42-44, 85] (4 Studien; 84 Patienten), koronarer Herzkrankheit [42] (1 Studie; 54 Patienten), kongestiver Herzinsuffizienz [33, 54] (2 Studien; 10 Patienten), Dyslipidämie [33, 54] (2 Studien; 11 Patienten) und ischämischer Kardiomyopathie [33]
(1 Studie; 3 Patienten) ein. Studien mit ILD-Patienten schlossen Patienten mit arterieller Hypertonie [196, 203, 207-209] (5 Studien; 49 Patienten), koronarer Herzkrankheit [207-209] (3 Studien; 21 Patienten), kongestiver Herzinsuffizienz [203] (1 Studie; 2 Patienten), pulmonaler Hypertonie [207-209] (3 Studien; 15 Patienten) und einer Herzerkrankung in der Vorgeschichte [198] (1 Studie; 1 Patient) ein. Von diesen enthielten nur 3 Studien [196, 207, 209], an denen Patienten mit ILD teilnahmen, Angaben zu kardiovaskulären Outcomes und Outcome-Maßen.

Neunzehn Studien mit COPD-Patienten [37, 49, 53, 57, 80, 90, 104, 106-108, 120, 121, 123, 124, 128, 129, 136, 146, 147], 2 Studien mit 

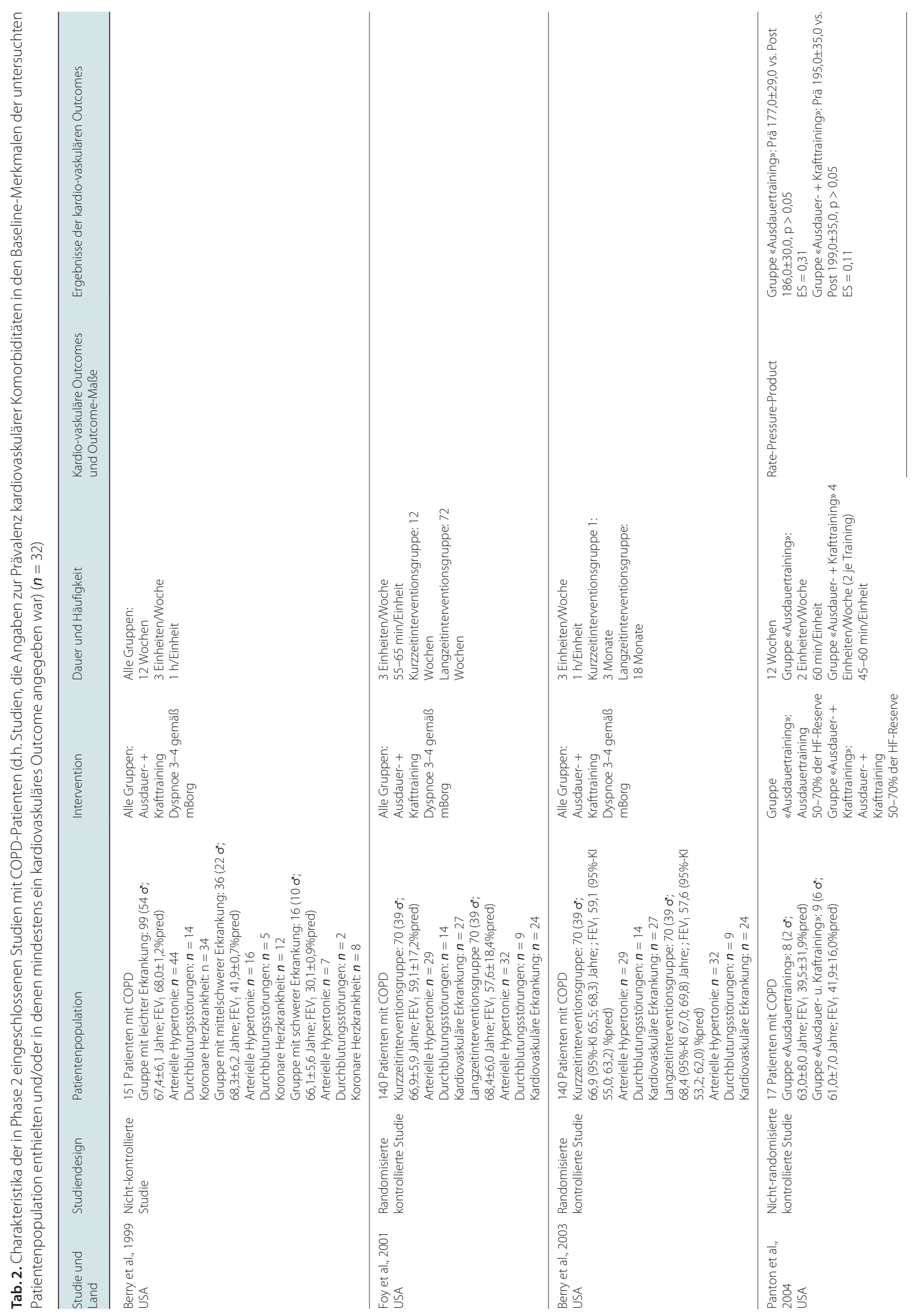


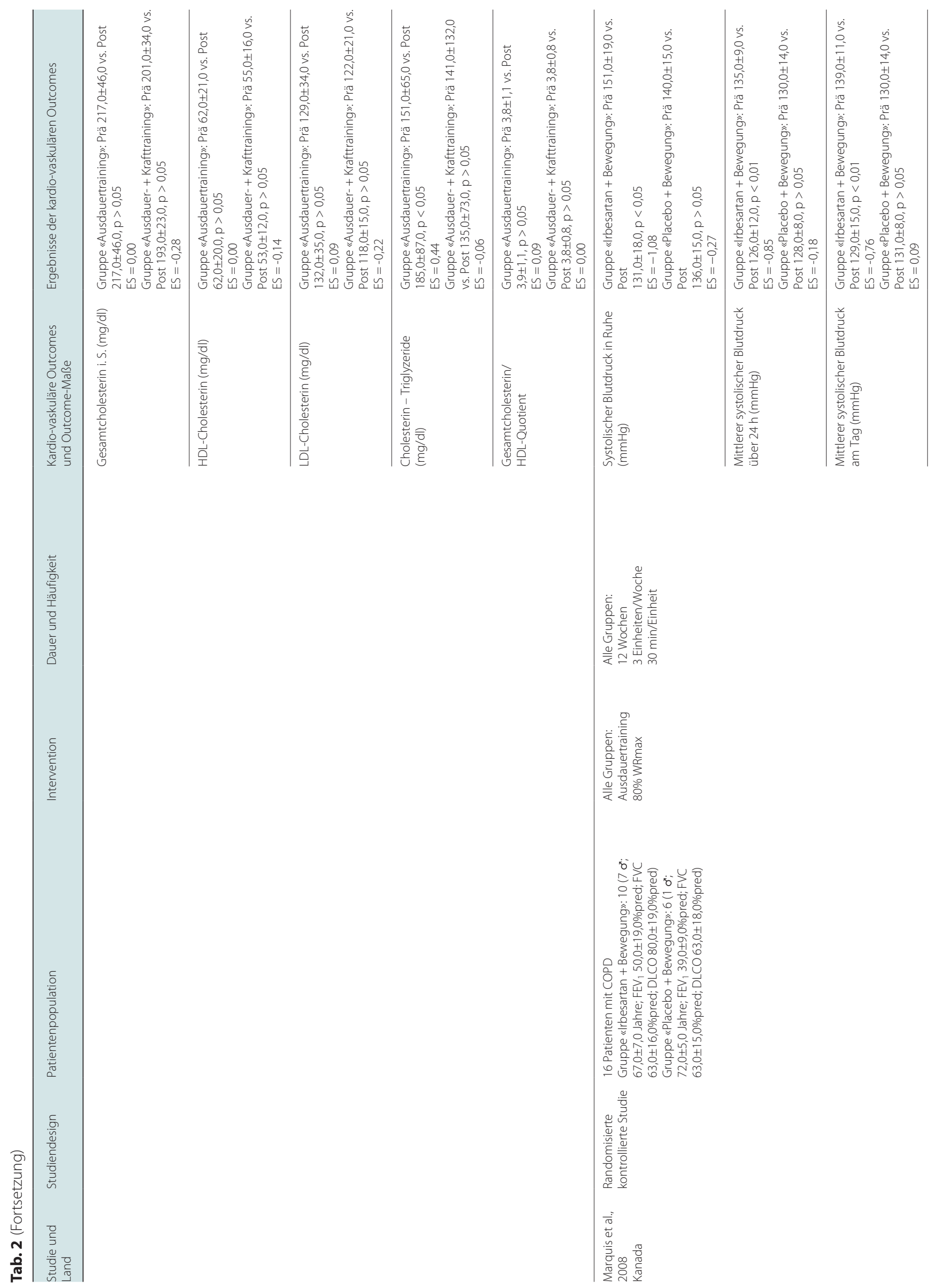

Kompass Pneumol 2020;8:178-199 


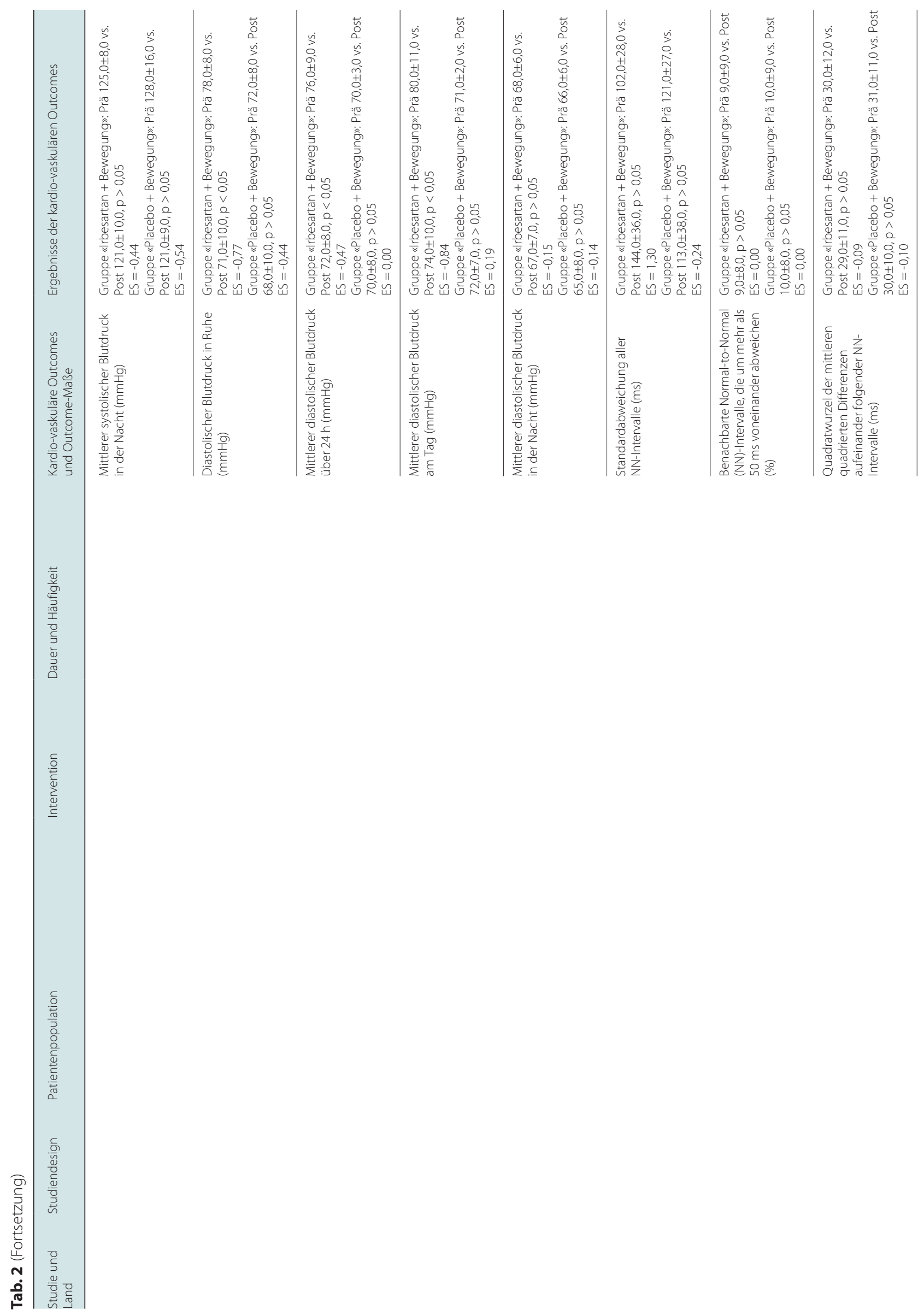




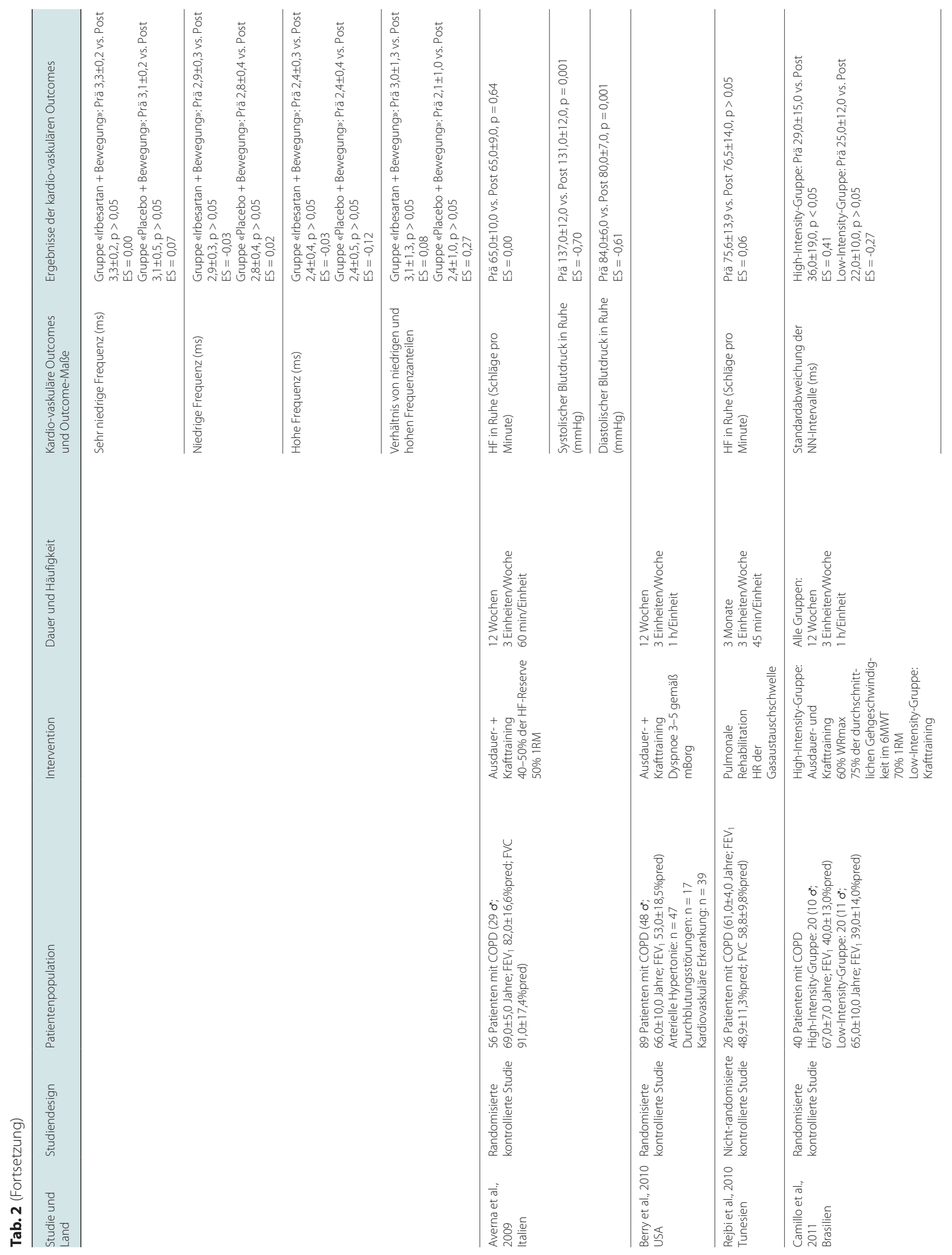




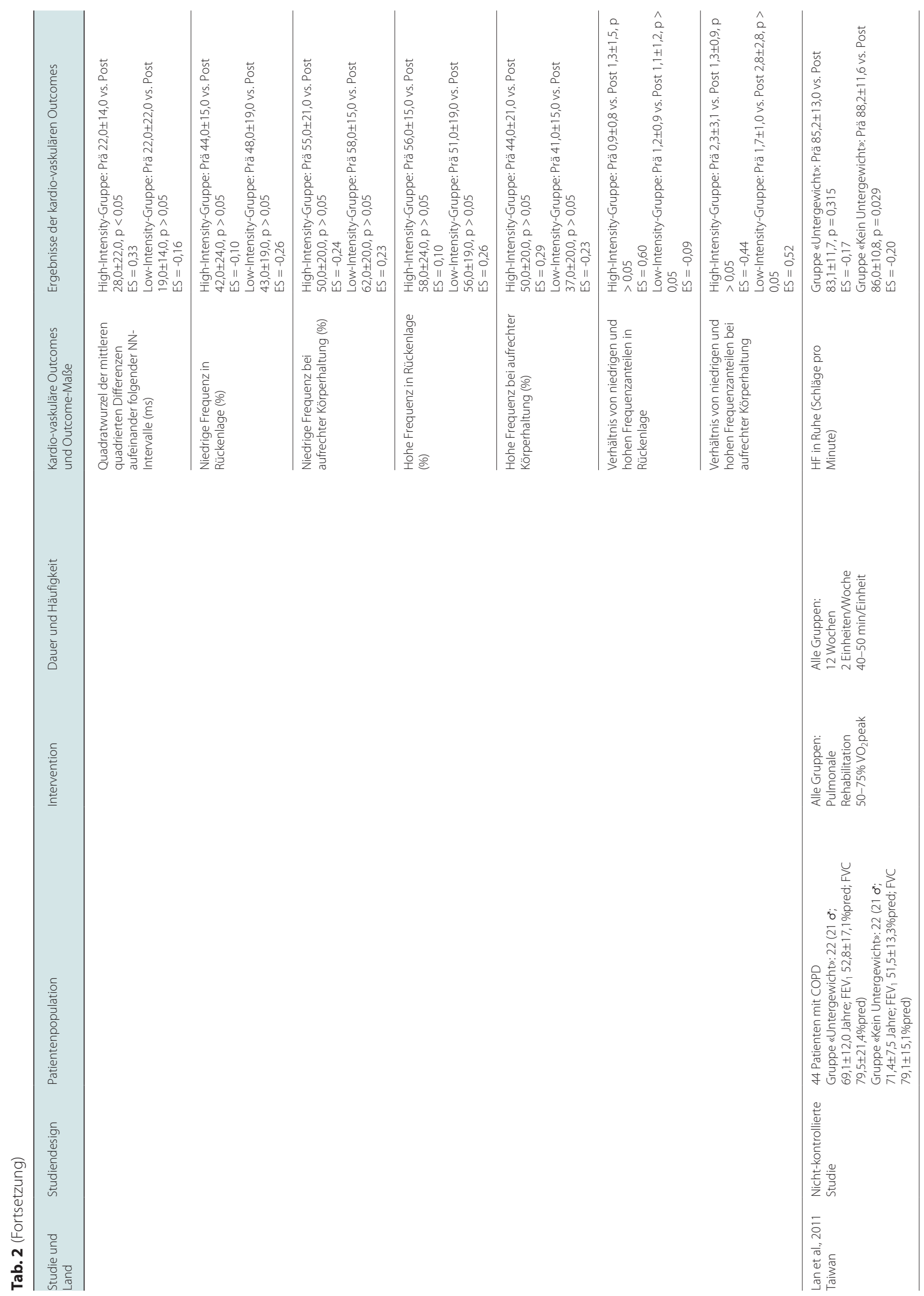



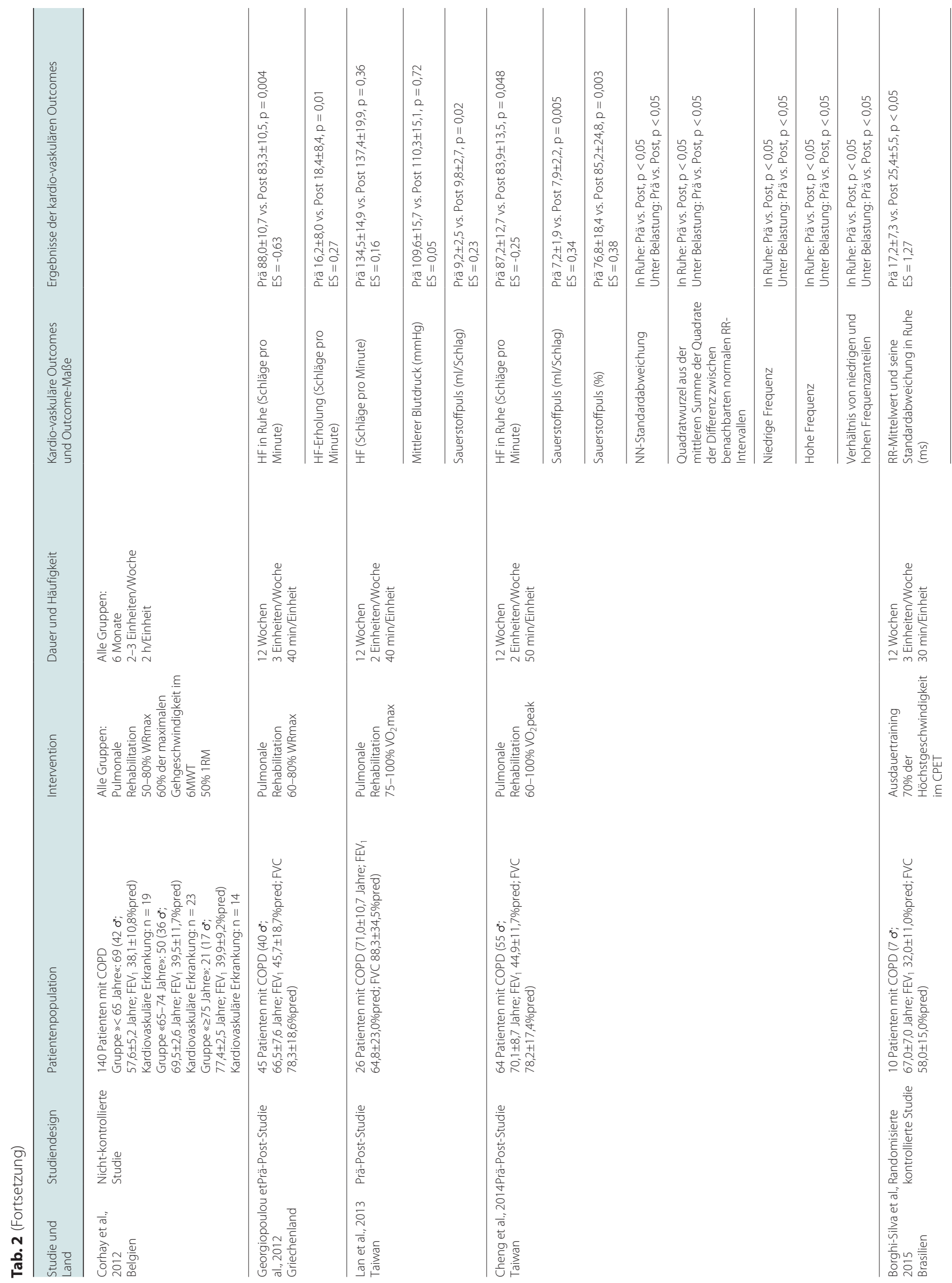

Kompass Pneumol 2020;8:178-199 


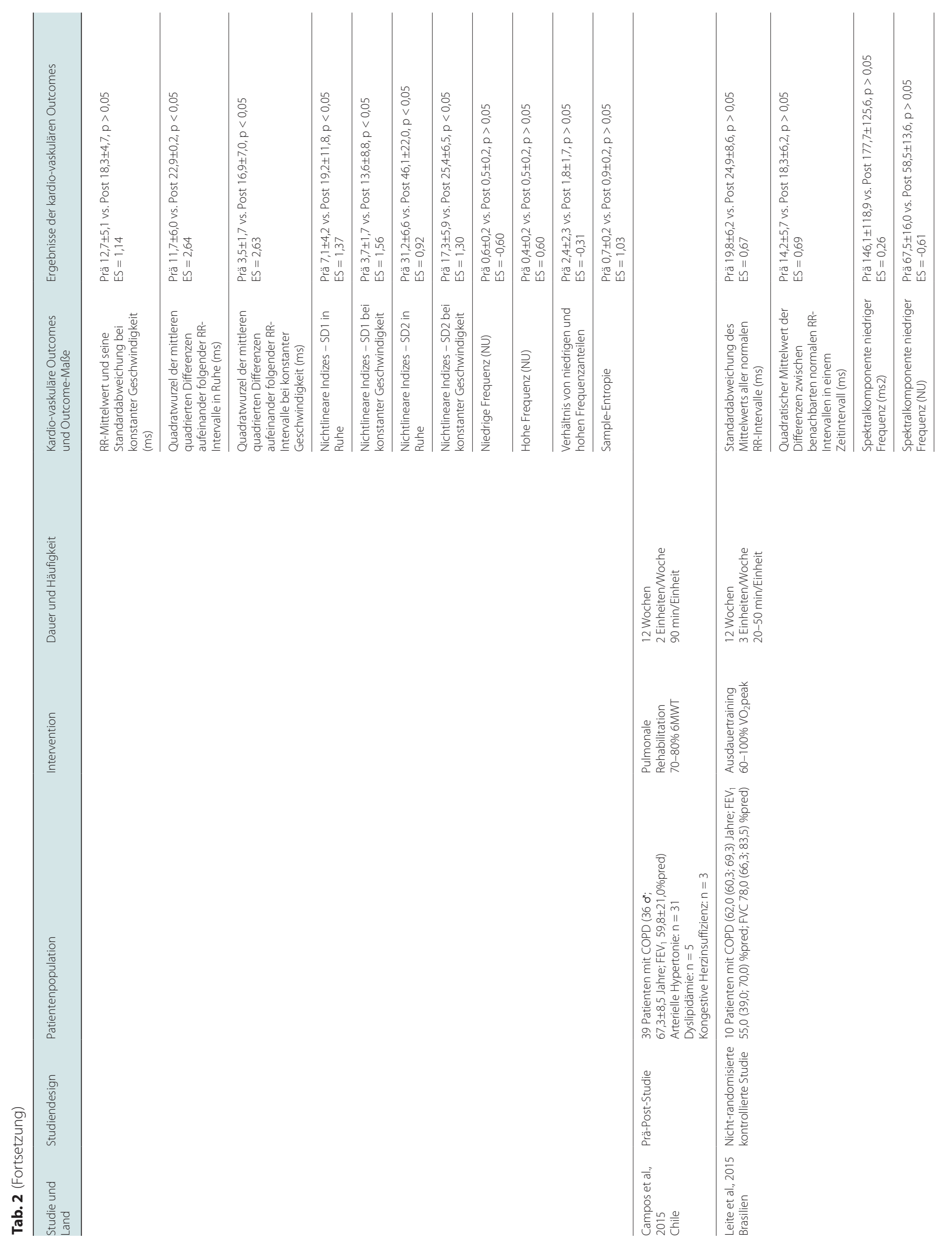




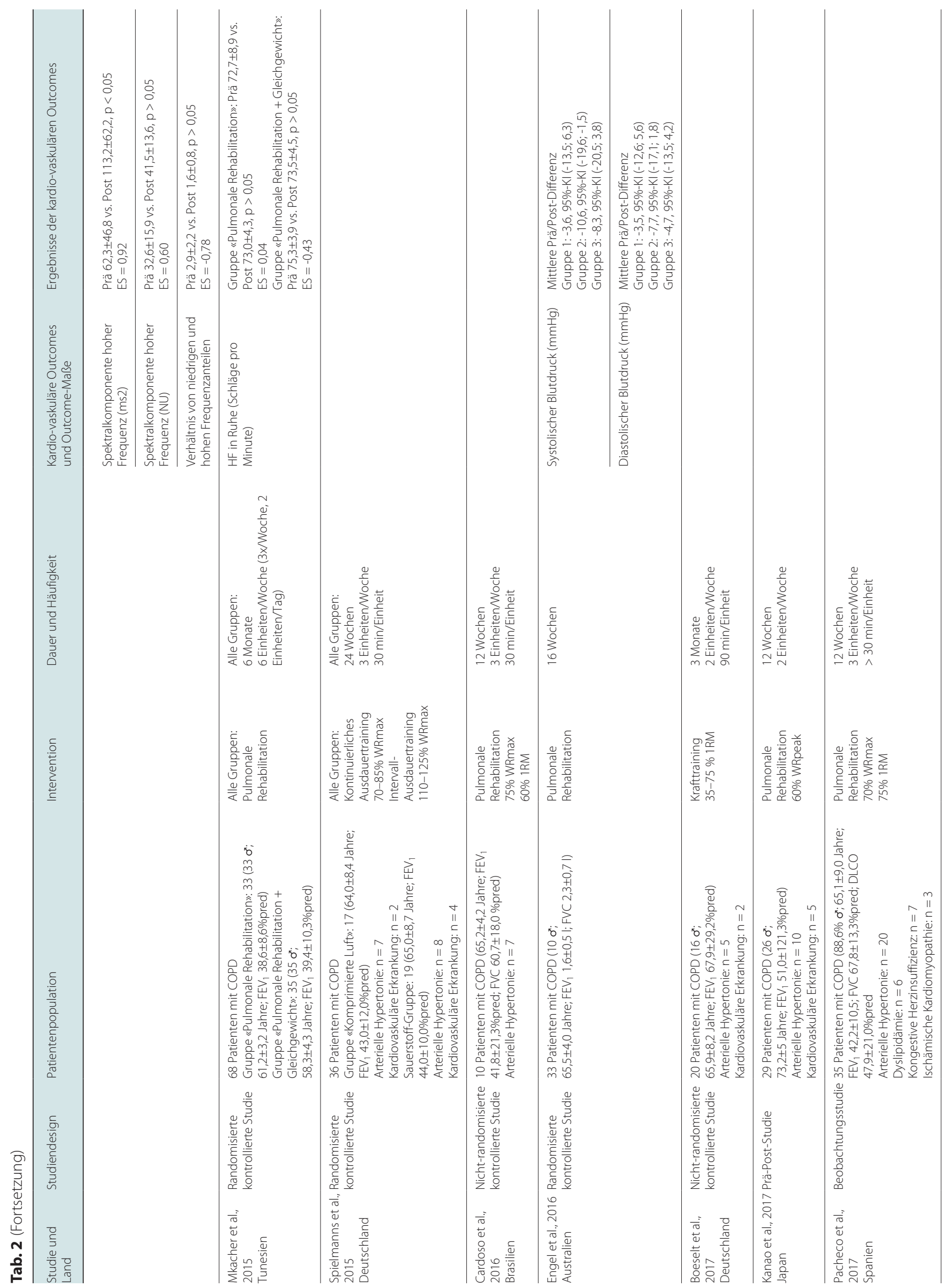




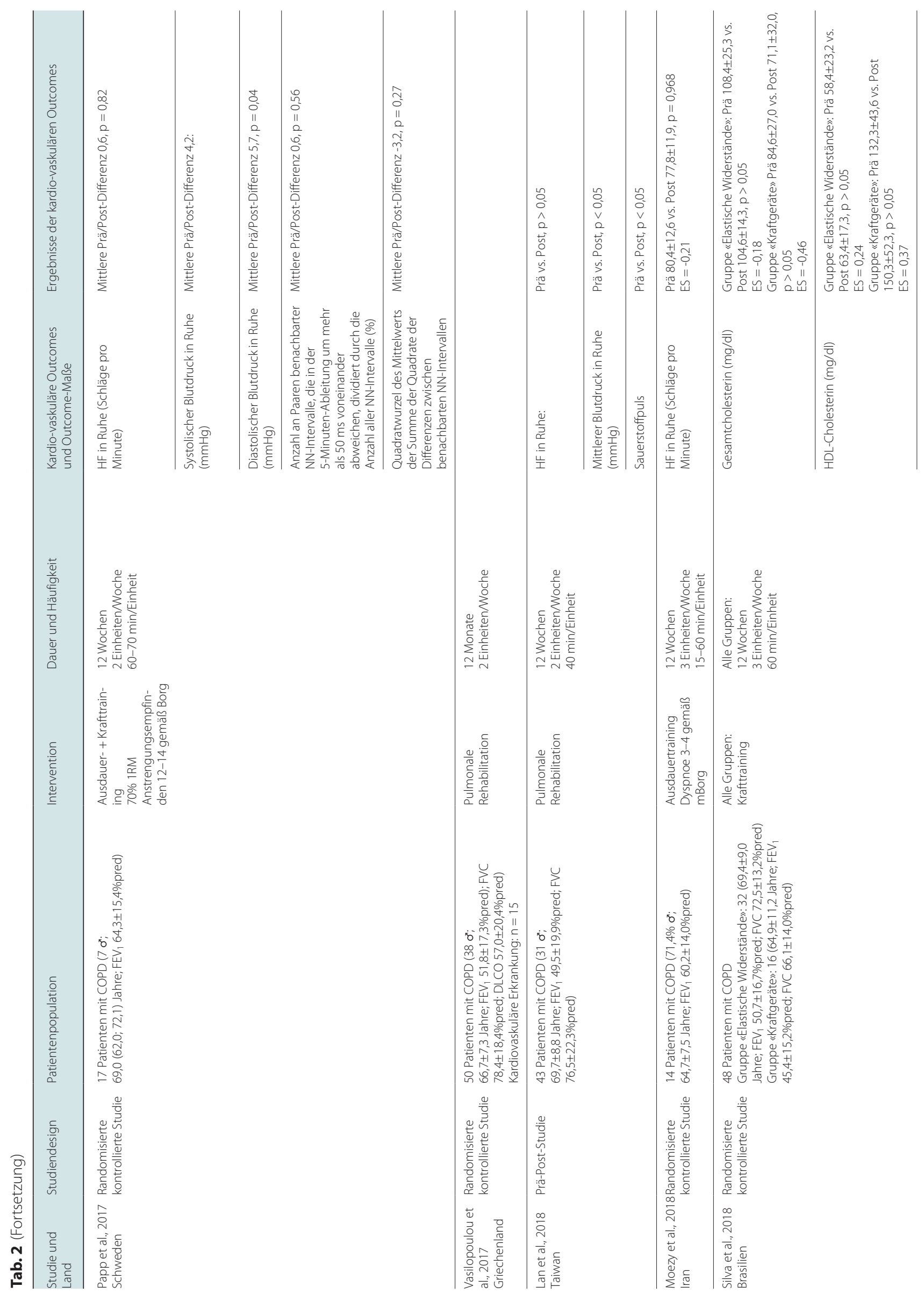




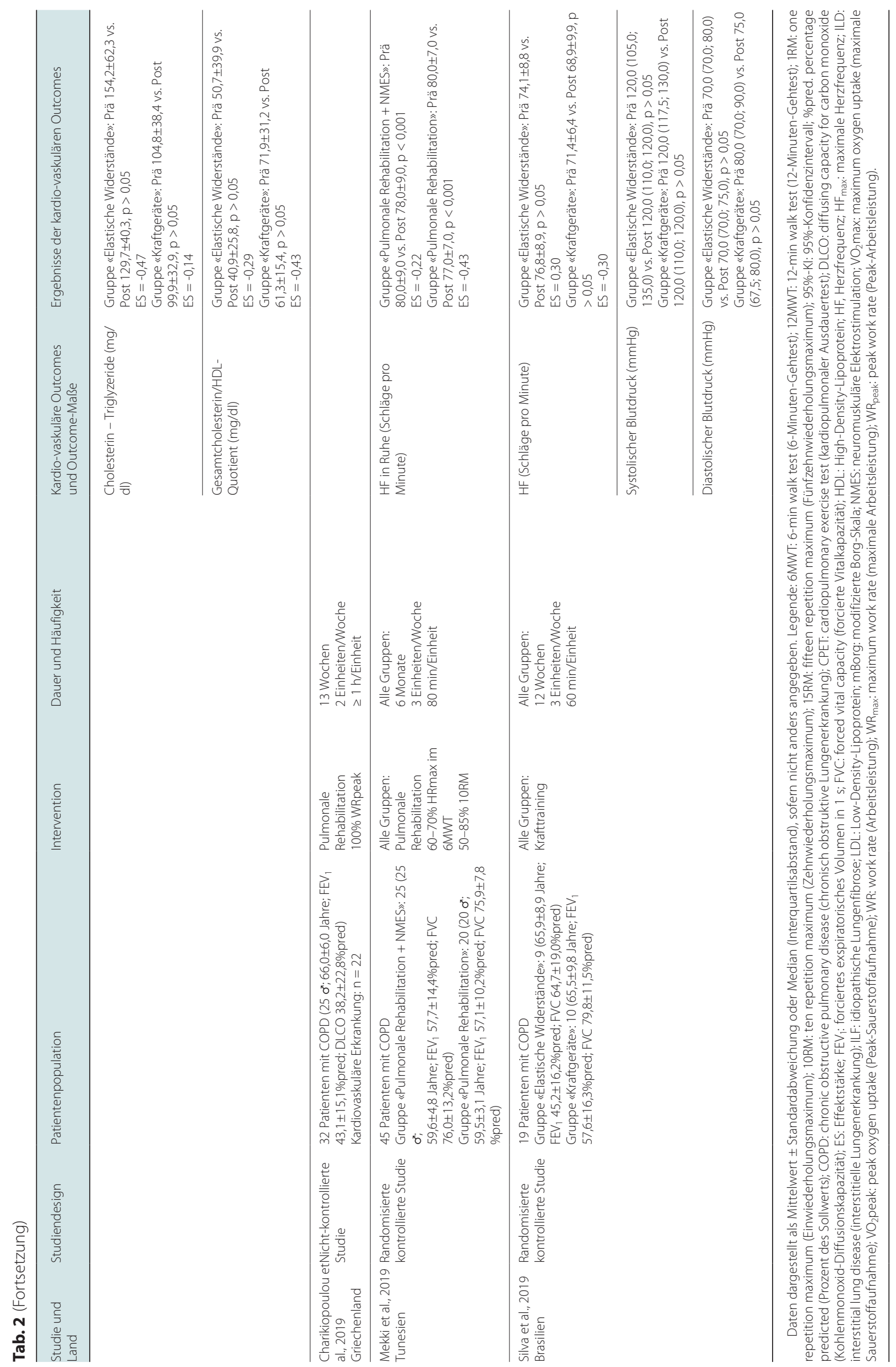


Tab. 3. Charakteristika der in Phase 2 eingeschlossenen Studien mit Asthma-Patienten (d.h. Studien, die Angaben zur Prävalenz kardiovaskulärer Komorbiditäten in den Baseline-Merkmalen der untersuchten Patientenpopulation enthielten und/oder in denen mindestens ein kardiovaskuläres Outcome angegeben war) $(n=2)$

\begin{tabular}{|c|c|c|c|c|c|c|}
\hline $\begin{array}{l}\text { Studie und } \\
\text { Land }\end{array}$ & Studiendesign & Patientenpopulation & Intervention & $\begin{array}{l}\text { Dauer und } \\
\text { Häufigkeit }\end{array}$ & $\begin{array}{l}\text { Kardio-vaskuläre } \\
\text { Outcomes und } \\
\text { Outcome-Maße }\end{array}$ & $\begin{array}{l}\text { Ergebnisse der kardio- } \\
\text { vaskulären Outcomes }\end{array}$ \\
\hline \multirow[t]{4}{*}{$\begin{array}{l}\text { Cochrane } \\
\text { et al., } 1990 \\
\text { Schottland }\end{array}$} & \multirow[t]{4}{*}{$\begin{array}{l}\text { Randomisierte } \\
\text { kontrollierte } \\
\text { Studie }\end{array}$} & \multirow[t]{4}{*}{$\begin{array}{l}18 \text { Patienten mit } \\
\text { Asthma }(27,0 \pm 17,0 \\
\text { Jahre; } \mathrm{FEV}_{1} \\
76,0 \pm 12,0 \% \text { pred })\end{array}$} & \multirow[t]{4}{*}{$\begin{array}{l}\text { Ausdauer- und } \\
\text { Krafttraining } \\
\text { 75\% HFmax }\end{array}$} & \multirow[t]{4}{*}{$\begin{array}{l}3 \text { Monate } \\
3 \text { Einheiten/Woche } \\
30 \text { min/Einheit }\end{array}$} & $\begin{array}{l}\text { Sauerstoffpuls (ml/ } \\
\text { Schlag) }\end{array}$ & $\begin{array}{l}\text { Prä } 8,8 \pm 2,3 \text { vs. Post } \\
10,8 \pm 2,4, p<0,001 \\
E S=0,85\end{array}$ \\
\hline & & & & & $\begin{array}{l}\text { Gesamt Cholesterin i. S. } \\
(\mathrm{mmol} / \mathrm{l})\end{array}$ & $\begin{array}{l}\text { Prä } 5,4 \pm 1,1 \text { vs. Post } \\
5,3 \pm 1,1, p>0,05 \\
E S=-0,09\end{array}$ \\
\hline & & & & & $\begin{array}{l}\text { HDL-Cholesterin } \\
(\mathrm{mmol} / \mathrm{l})\end{array}$ & $\begin{array}{l}\text { Prä } 1,7 \pm 0,4 \text { vs. Post } \\
1,6 \pm 0,3, p>0,05 \\
E S=-0,28\end{array}$ \\
\hline & & & & & $\begin{array}{l}\text { LDL-Cholesterin } \\
(\mathrm{mmol} / \mathrm{l})\end{array}$ & $\begin{array}{l}\text { Prä } 3,2 \pm 1,2 \text { vs. Post } \\
2,9 \pm 0,9, p>0,05 \\
E S=-0,28\end{array}$ \\
\hline \multirow[t]{3}{*}{$\begin{array}{l}\text { El-Kader } \\
\text { et al., } 2016 \\
\text { Saudi-Arabien }\end{array}$} & \multirow[t]{3}{*}{$\begin{array}{l}\text { Randomisierte } \\
\text { kontrollierte } \\
\text { Studie }\end{array}$} & \multirow[t]{3}{*}{$\begin{array}{l}40 \text { Patienten mit } \\
\text { Asthma }\left(23 \sigma^{2} ; 47,2 \pm 6,5\right. \\
\text { Jahre; FEV } 1,4 \pm 0,7 \text { I) }\end{array}$} & \multirow[t]{3}{*}{$\begin{array}{l}\text { Ausdauertraining } \\
\text { 60-80\% HFmax }\end{array}$} & \multirow[t]{3}{*}{$\begin{array}{l}6 \text { Monate } \\
3 \text { Einheiten/Woche } \\
30 \text { min/Einheit }\end{array}$} & $\begin{array}{l}\text { HDL-Cholesterin } \\
(\mathrm{mg} / \mathrm{dl})\end{array}$ & $\begin{array}{l}\text { Prä } 34,7 \pm 5,6 \text { vs. Post } \\
37,9 \pm 4,6, p<0,05 \\
E S=0,62\end{array}$ \\
\hline & & & & & $\begin{array}{l}\text { LDL-Cholesterin } \\
(\mathrm{mg} / \mathrm{dl})\end{array}$ & $\begin{array}{l}\text { Prä } 133,7 \pm 13,2 \text { vs. Post } \\
120,3 \pm 11,5, p<0,05 \\
E S=-1,08\end{array}$ \\
\hline & & & & & $\begin{array}{l}\text { Cholesterin - } \\
\text { Triglyzeride (mg/dl) }\end{array}$ & $\begin{array}{l}\text { Prä } 155,4 \pm 12,6 \text { vs. Post } \\
127,7 \pm 11,3, p<1,05 \\
E S=-2,31\end{array}$ \\
\hline
\end{tabular}

Daten dargestellt als Mittelwert土Standardabweichung, sofern nicht anders angegeben. Legende: \%pred: percentage predicted (Prozent des Sollwerts); ES: Effektstärke; FEV : forciertes exspiratorisches Volumen in 1 s; HDL: High-Density-Lipoprotein; HF: Herzfrequenz; HF max: maximale Herzfrequenz; LDL: LowDensity-Lipoprotein.

Asthma-Patienten [186, 189] und 5 Studien mit ILD-Patienten [196, 201, 202, 207, 209] enthielten Angaben zu kardiovaskulären Outcomes und Outcome-Maßen. In Studien mit COPD-Patienten fand sich eine Vielzahl von Outcome-Maßen, wohingegen Studien mit Asthma-Patienten hauptsächlich auf das Serumlipidprofil ausgerichtet waren $[187,190](\mathrm{n}=2)$, und in Studien mit ILD-Patienten vornehmlich die Herzfrequenz in Ruhe [196, 201, 209] ( $\mathrm{n}=3$ ) und der Blutdruck [196, 207, 209] ( $=3$ ) berichtet wurden. Das am häufigsten berichtete Outcome-Maß war die Herzfrequenz in Ruhe $[37,57,90,106,107,121,123,124,129,136$, $196,201,209](\mathrm{n}=13$; Effektstärke (effect size, ES) $=[-0,63 ; 0,11])$. In den meisten Studien ( $\mathrm{n}=20 ; 71,4 \%)$ waren nur geringe bis moderate Effekte bei den berichteten kardiovaskulären OutcomeMaßen zu beobachten. Die Standardabweichung der RR-Intervalle $[49,108](\mathrm{n}=2$; $\mathrm{ES}=[0,67 ; 2,64])$ und der quadratische Mittelwert der Differenzen aufeinander folgender RR-Intervalle $[49,57,108](\mathrm{n}=3$; ES $=[0,69 ; 2,64])$ waren die Outcome-Maße, die stärkere Effekte zeigten. Bei Patienten mit COPD führten die Effekte des Bewegungsprogramms auf die Herzfrequenz in Ruhe zu einer gepoolten Gesamt-Effektstärke von -0,23 (95\%-KI) -0,33 bis -0,13) (Abb. 5).

In Bezug auf die Bewegungsprogramme beinhalteten die meisten Studien mit COPD-Patienten ein pulmonales Rehabilitationspro- gramm $[33,54,55,57,59,68,80,90,101,104,106,107,121,123$, $136,167](\mathrm{n}=16)$ oder ein kombiniertes Bewegungsprogramm aus Ausdauer- und Krafttraining [38, 43-45, 54, 86, 129, 130] (n $=8$ ). Die Trainingseinheiten erfolgten 2 bis $6 \mathrm{Mal}$ pro Woche und dauerten jeweils zwischen 15 Minuten und 2 Stunden. Die Programmdauer betrug zwischen 12 Wochen und 18 Monaten. Die verordneten Bewegungsprogramme wiesen ein breites Intensitätsspektrum auf: $60 \%$ bis $80 \%$ der maximalen Herzfrequenz, $50 \%$ bis $100 \%$ der Peak- oder maximalen Sauerstoffaufnahme, $50 \%$ bis $125 \%$ der Peak- oder maximalen Arbeitsbelastung, 35\% bis $75 \%$ des Einer-Wiederholungs-Maximums, Dyspnoe und Anstrengungsempfinden zwischen 3 und 6 auf der modifizierten Borg-Skala und 12 bis 16 auf der Borg-Skala. Keine der Studien enthielt Angaben über etwaige Anpassungen der Bewegungsprogramme für Patienten mit kardiovaskulären Komorbiditäten. Nur eine Studie [108] berichtete über eine Anpassung des Bewegungsprogramms in verschiedenen Mesozyklen, um bestimmte kardiovaskuläre Outcomes zu verbessern.

In Studien mit Asthma-Patienten erfolgte ein dreimonatiges kombiniertes Bewegungsprogramm aus Ausdauer- und Krafttraining [189] oder ein sechsmonatiges Ausdauertraining [186]. Die Trainingseinheiten fanden $3 \mathrm{Mal}$ pro Woche statt und dauerten jeweils 30 Minuten. Die Intensität lag bei $60 \%$ bis $80 \%$ der
194

Kompass Pneumol 2020;8:178-199 DOI: $10.1159 / 000509305$ 


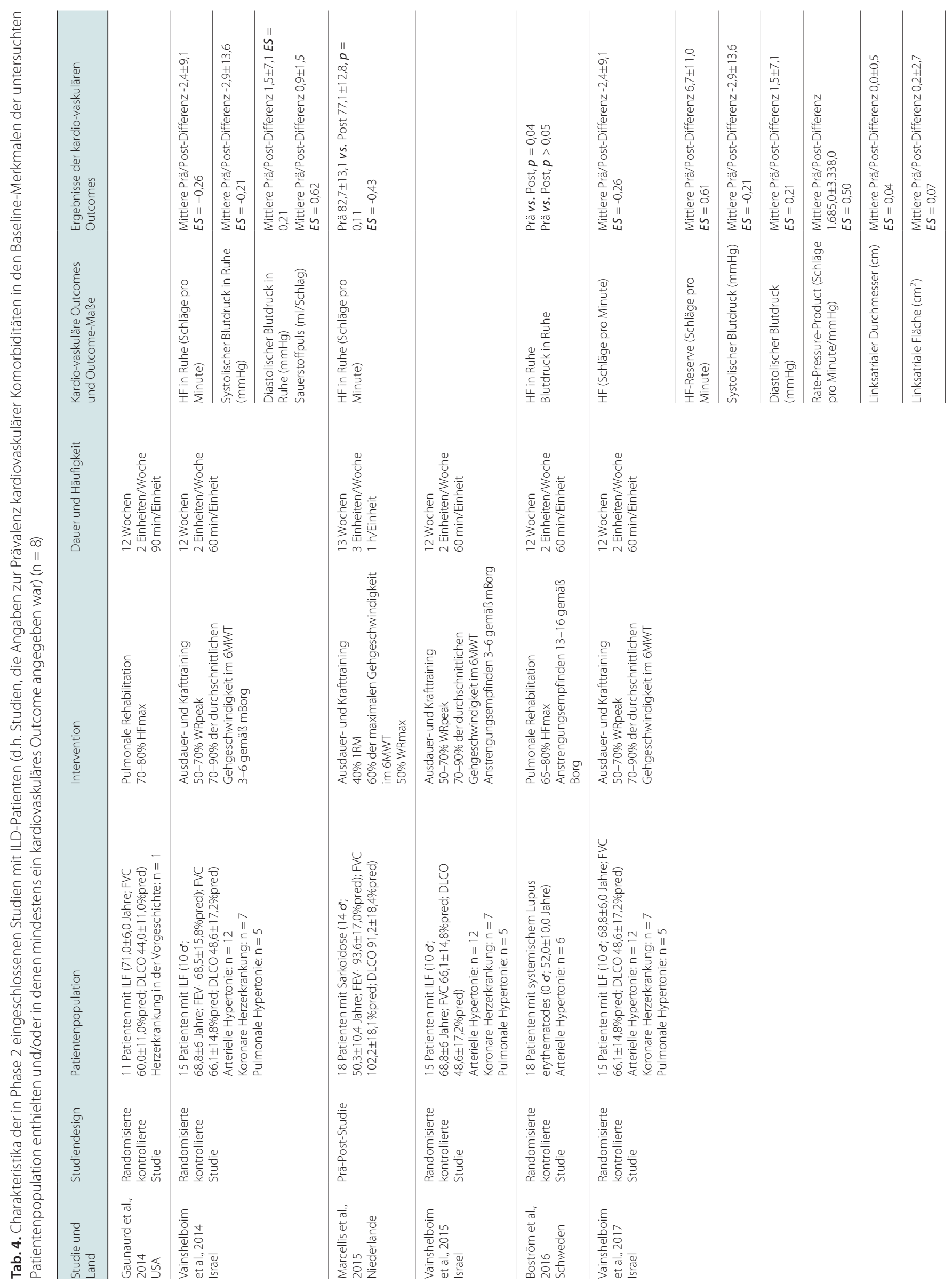




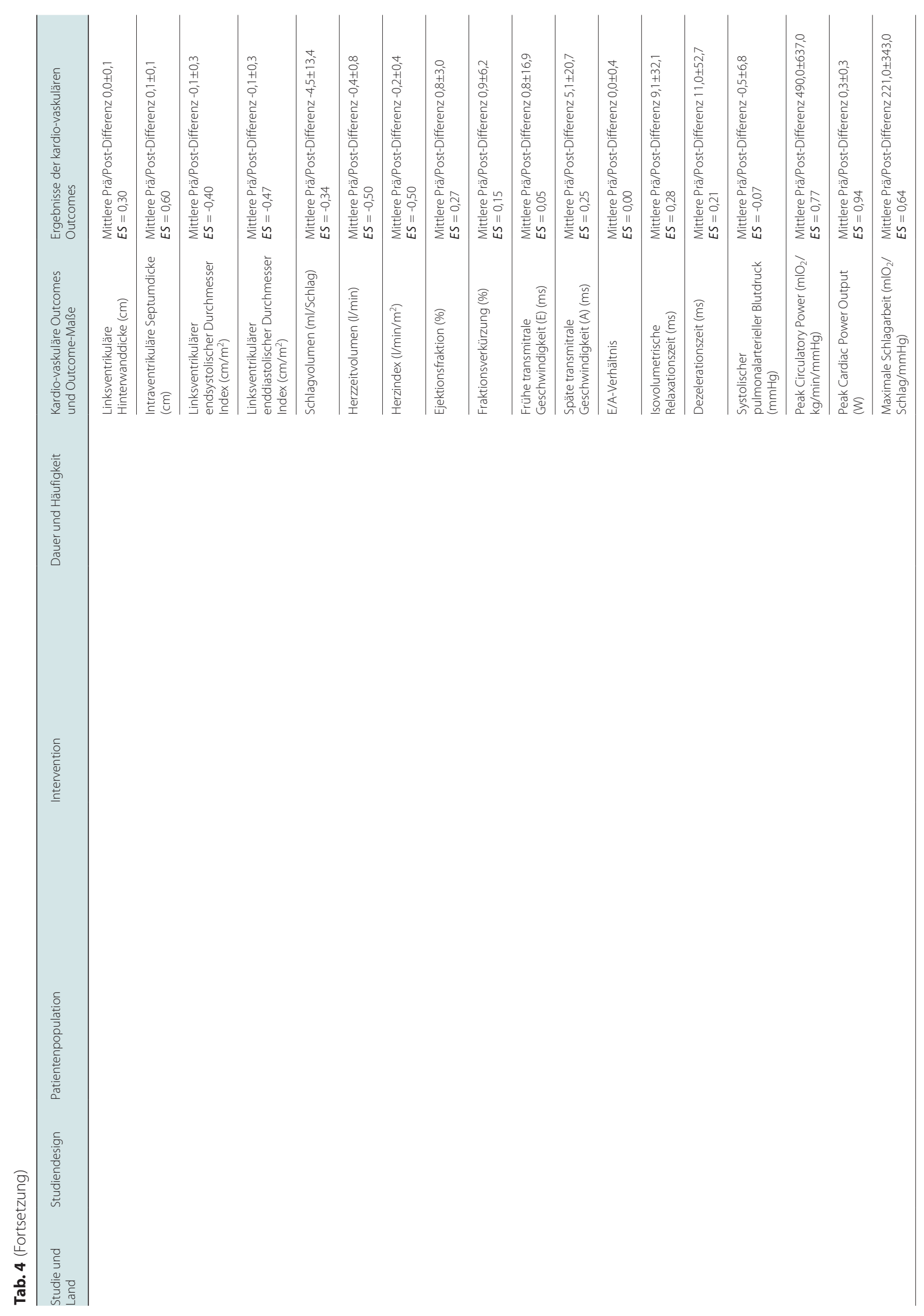


maximalen Herzfrequenz. Spezielle Anpassungen zur Verbesserung bestimmter kardiovaskulärer Outcomes wurden nicht berichtet.

In den meisten Studien mit ILD-Patienten erfolgte ein kombiniertes Bewegungsprogramm aus Ausdauer- und Krafttraining [201203, 207-209] $(\mathrm{n}=6)$. Der Großteil der Programme dauerte 12 Wochen mit 2 Trainingseinheiten pro Woche $[196,198,202,203$, 207-209] $(\mathrm{n}=7)$. Die Trainingseinheiten dauerten jeweils 60 bis 90 Minuten bei einer verordneten Intensität zwischen $65 \%$ und 85\% der maximalen Herzfrequenz, einer Peak-Arbeitsbelastung von $50 \%$ bis $90 \%$ und Dyspnoe und Anstrengungsempfinden zwischen 3 und 6 auf der modifizierten Borg-Skala. Keine der Studien enthielt Angaben über etwaige Anpassungen der Bewegungsprogramme für Patienten mit kardiovaskulären Komorbiditäten.

\section{Diskussion}

Nach bestem Wissen der Autoren handelt es sich bei der vorliegenden Arbeit um die erste umfassende Übersicht über die wissenschaftliche Literatur, die zusammenfasst, welche Eignungskriterien in Bezug auf kardiovaskuläre Erkrankungen herangezogen wurden, um Patienten mit chronischen Atemwegserkrankungen für Studien zu Bewegungsprogrammen auszuwählen und welche Auswirkungen ein mindestens dreimonatiges Bewegungstraining auf die kardiovaskulären Outcomes hat sowie welche Anpassungen vorgenommen wurden, um die verordneten Bewegungsprogramme auf Patienten mit kardiovaskulären Komorbiditäten zuzuschneiden. Dabei zeigte sich zum einen, dass im Großteil der Studien (58,9\%) Patienten mit kardiovaskulären Komorbiditäten a priori ausgeschlossen waren, zum anderen, dass die Evidenzlage über die Auswirkungen von Bewegungstraining auf die kardiovaskulären Outcomes von Patienten mit chronischen Atemwegserkrankungen begrenzt ist und keine der Studien explizite Angaben enthielt, wie die Trainingsmodalitäten in Hinblick auf kardiovaskuläre Komorbiditäten angepasst werden können.

Das Spektrum der kardiovaskulären Erkrankungen, die in trainingsbezogenen wissenschaftlichen Untersuchungen Ausschlusskriterien bilden, war breit. Interessanterweise gilt die Mehrzahl der berichteten Ausschlusskriterien (34/45) nicht als Kontraindikation für Bewegungstraining. Tatsächlich schlossen wenige Studien $(18,3 \%)$ nur Patienten mit akuter/instabiler kardiovaskulärer Erkrankung, die eine Kontraindikation für die Teilnahme an einem Bewegungstraining darstellt, aus. Die meisten Studien schlossen Patienten sowohl mit stabilen als auch mit instabilen kardiovaskulären Komorbiditäten aus, obwohl mindestens $20 \%$ bis 50\% der Patienten mit COPD, Asthma oder ILD kardiovaskuläre Komorbiditäten aufweisen [5, 7, 18, 20, 210, 211]. Werden Patienten mit kardiovaskulären oder anderen Komorbiditäten, die keine Kontraindikation für ein Bewegungstraining darstellen, ausgeschlossen, hat dies zur Folge, dass die gewonnenen Erkenntnisse nur für eine Untergruppe oder in einigen Fällen sogar nur für eine Minderheit der Patienten in die klinische Praxis übertragen werden können. Dies könnte weitreichende Kon- 


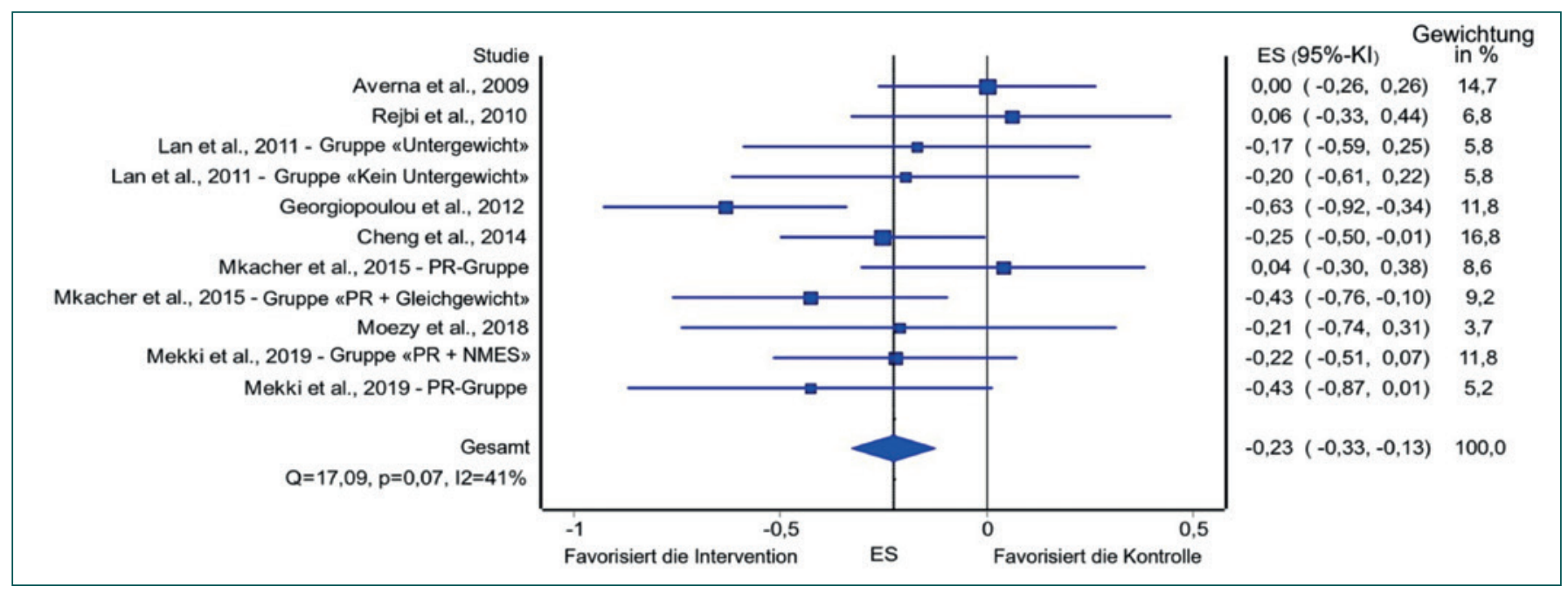

Abb. 5. Forest-Plot des Vergleichs «Kontrolle vs. Intervention» bei Patienten mit COPD; Outcome: Herzfrequenz in Ruhe. ES: Effektstärke; NMES: neuromuskuläre Elektrostimulation; PR: pulmonale Rehabilitation.

sequenzen haben, da das aktuelle Wissen (einschließlich der klinischen Leitlinien) krankheitszentriert ist und daher nicht ausreicht, um Kliniker entsprechend zu unterstützen oder anzuleiten, wie Bewegungsprogramme für Patienten mit chronischen Atemwegserkrankungen und multiplen chronischen Erkrankungen zu verordnen sind $[13,212]$. Zudem waren einige der berichteten Kriterien (z.B. kardiovaskuläre Erkrankung) zu vage, um nachzuvollziehen, welche Erkrankungen tatsächlich ausgeschlossen wurden, und mehr als 20\% der eingeschlossenen Studien enthielten keine Angaben zu den Auswahlkriterien, obwohl diese Informationen von zentraler Bedeutung sind, um Klarheit und Transparenz der Forschungsarbeit zu gewährleisten [213].

Bewegungsprogramme führten bei Patienten mit kardiovaskulären Komorbiditäten zu ähnlichen signifikanten Verbesserungen der berichteten allgemeinen Outcomes, d.h. der Symptome, der Funktionsfähigkeit, der Belastungskapazität, der Muskelkraft und der gesundheitsbezogenen Lebensqualität, wie sie normalerweise bei Patienten mit Atemwegserkrankungen zu beobachten sind [22]. In Bezug auf die kardiovaskulären Outcomes wurden in den meisten Studien $(71,4 \%)$ jedoch nur geringe bis mäßige Effekte gefunden, wobei bei den Maßen der Herzfrequenzvariabilität $(\mathrm{ES}=[-0,78 ; 2,64])$ und des Serumlipidprofils $(\mathrm{ES}=[-2,31 ; 0$, 62]) größere Effekte berichtet wurden. Daneben war ein leichter, aber signifikanter Gesamteffekt der Bewegungsprogramme auf die Ruheherzfrequenz von Patienten mit COPD nachweisbar. Diese Ergebnisse sind gegenüber den zuvor berichteten Ergebnissen für Patienten mit Kardiovaskulären Erkrankungen, bei denen positive Effekte des Bewegungstrainings auf die Herzfrequenzvariabilität und die Erholung der Herzfrequenz zu beobachten waren, nicht unterlegen [214], und für den arteriellen Blutdruck sowie das Serumlipidprofil wurden uneinheitliche, aber signifikante und bescheidene Effekte berichtet [214-217]. Unserer Vermutung nach könnten mehrere Gründe zu den gefundenen begrenzten Wirkungen beitragen. Zum einen enthielten die meis- ten Studien keine Angaben zu speziellen Anpassungen der verordneten Bewegungsprogramme für Patienten mit kardiovaskulären Komorbiditäten, doch kann es durchaus sein, dass die Anpassungen erfolgten, ohne dass in der Veröffentlichung speziell darüber berichtet wurde. Man weiß, dass kardiovaskuläre Erkrankungen ein besonderes Augenmerk bei der Erstellung des Trainingsplans erfordern [22], und je nach Art und Schweregrad der vorliegenden kardiovaskulären Erkrankung (z.B. koronare Herzkrankheit, kongestive Herzinsuffizienz, periphere arterielle Verschlusskrankheit, pulmonale arterielle Hypertonie) existieren unterschiedliche Empfehlungen [218]. Tatsächlich müssen Dauer, Häufigkeit, Modus, Intensität und Überwachung des Trainings an die Besonderheiten und Bedürfnisse des Patienten, seine klinischen Erkrankungen, den kardiovaskulären Phänotyp (Risikofaktoren und Krankheiten), den Fitness-Level, die Medikamenteneinnahme (Betablocker, Statine, Glinide, Sulfonylharnstoff), abnorme Reaktionen auf Belastung (Myokardischämie, Vorhofflimmern, ventrikuläre Tachykardie) und die angestrebten Rehabilitationsziele angepasst werden [1, 218-221]. Außerdem hängt der Effekt des Bewegungstrainings vom richtigen Zuschnitt des Trainingsprogramms ab, da es sich gezeigt hat, dass unterschiedliche Trainingsverordnungen mit signifikant unterschiedlichen klinischen Outcomes verbunden sind [221]. Zukünftige Studien sollten daher den Effekt von Bewegungsprogrammen, die speziell auf Patienten mit gleichzeitig bestehenden Atemwegserkrankungen und kardiovaskulären Komorbiditäten zugeschnitten sind, bewerten [7] und in allen Einzelheiten über die Intervention berichten. Ferner empfehlen die Leitlinien für kardiale Rehabilitation der führenden wissenschaftlichen Gesellschaften, das Training dreimal wöchentlich durchzuführen und die Intensität von moderat zu stark zu steigern [21]. In einigen der eingeschlossenen Studien erfolgte das Training jedoch bei geringerer Intensität und/oder weniger häufig pro Woche, und dies könnte ebenfalls zum relativen Mangel an Effekt beigetragen haben, weil die Min- 
destbelastungsdosis für einen kardiovaskulären Nutzen (>150 min/Woche Ausdauertraining, Energieverbrauch 1000-2000 kcal/Woche) möglicherweise nicht erreicht wurde [218, 222]. Zudem beinhalteten einige der eingeschlossenen Studien nur Krafttraining als Trainingsprogramm. Tatsächlich wird Krafttraining für Patienten mit kardiovaskulären Erkrankungen empfohlen, allerdings zusätzlich zum Ausdauertraining, das eine zentrale Komponente in der Rehabilitation dieser Patienten bildet [21]. Diese Beobachtungen zeigten, dass die derzeitige Verordnung von Bewegungsprogrammen für Patienten mit COPD, Asthma oder ILD und kardiovaskulären Komorbiditäten bei weitem nicht optimal ist und gründlich überdacht werden muss. Digitale Unterstützung für die Verordnung von Bewegungsprogrammen für diese Patienten entsprechend den verschiedenen klinischen Leitlinien für unterschiedliche kardiovaskuläre Erkrankungen ist jedoch verfügbar und kann daher zur Unterstützung der Ärzte eingesetzt werden [218]. Und schließlich waren die meisten Studien, die Patienten mit kardiovaskulären Komorbiditäten einschlossen, nur auf die Beurteilung der Herzfrequenz in Ruhe ausgerichtet. Zwar handelt es sich dabei um ein relevantes Outcome-Maß und die Ergebnisse der Metaanalyse bei COPD-Patienten fallen zugunsten einer Intervention aus, doch plädieren die Empfehlungen für Patienten mit kardiovaskulären Erkrankungen für eine umfassendere Beurteilung, bei der auch Outcomes wie arterieller Blutdruck, Serumlipidprofil oder die Ergebnisse der Echokardiographie, die den Zielen der Rehabilitation bei diesen Patienten besser entsprechen, berücksichtigt werden [223, 224]. Daher sollten die Outcomes besser auf die kardiovaskulären Komorbiditäten der Patienten ausgerichtet werden [19].

Trotz der bekannten Prävalenz und dem erhöhten Morbiditätsund Mortalitätsrisiko, das kardiovaskuläre Komorbiditäten für Patienten mit chronischen Atemwegserkrankungen darstellen [7, 211], wurden nur in 3 Studien [196, 207, 209] (alle aus den vergangenen 5 Jahren) Patienten mit kardiovaskulären Komorbiditäten aufgenommen und eine Bewertung der kardiovaskulären Outcome-Maße durchgeführt. Dies verdeutlicht die derzeit in der Literatur bestehende Kluft in Bezug auf Bewegungsprogramme und zeigt, dass spezielle Studien erforderlich sind, die auf die kardiovaskulären Outcomes bei diesen Patienten ausgerichtet sind.

Die vorliegende systematische Übersichtsarbeit hat etliche Einschränkungen, die es zu berücksichtigen gilt. Zum einen wurden unter der Annahme, dass eine große Anzahl von Studien gefunden werden würde, nur Übungsprogramme mit einer Dauer von mindestens 12 Wochen eingeschlossen, wodurch möglicherweise andere relevante Studien verloren gegangen sind. Eine Mindestübungsdauer von 12 Wochen wird jedoch empfohlen, um bei Pa- tienten mit kardiovaskulären Erkrankungen einen Nutzen zu erzielen [25]. Aufgrund der Tatsache, dass nur wenige der gefundenen Studien Patienten mit ILD einschlossen, erfolgte zudem eine Gruppierung aller ILD-Arten, obwohl verschiedene Arten von ILD unterschiedliche Merkmale aufweisen und möglicherweise mit unterschiedlichen kardiovaskulären Komorbiditäten und Reaktionen auf Bewegungsprogramme verbunden sind. Und zum Dritten war die Mehrzahl der eingeschlossenen Studien von schwacher Qualität. Da eine Verblindung der Probanden bei Bewegungsinterventionen jedoch nicht möglich ist und die Patienten meist vom Arzt überwiesen werden, um ihre Sicherheit zu gewährleisten, war es praktisch unmöglich, eine hohe Qualität mit dem zur Qualitätsbeurteilung verwendeten Instrument sicherzustellen.

\section{Schlussfolgerungen}

Zwar untersuchten zahlreiche Studien die Effekte eines mindestens dreimonatigen Bewegungstrainings bei Patienten mit chronischen Atemwegserkrankungen, doch nur wenige Studien schlossen Patienten mit kardiovaskulären Komorbiditäten ein. Die festgestellten Effekte der Bewegungsprogramme auf die kardiovaskulären Outcome-Maße waren begrenzt, was daran liegen könnte, dass keine Anpassung des verordneten Bewegungsprogramms erfolgte und der Umfang der kardiovaskulären Outcome-Maße nicht ausreichend war. Um die bestehende Lücke in der Literatur zu schließen, sind zukünftige Studien erforderlich, die auf Patienten mit gleichzeitig vorliegenden respiratorischen und kardiovaskulären Erkrankungen ausgerichtet sind und die die Effekte von speziell auf diese Patienten zugeschnittenen Bewegungsprogrammen untersuchen.

\section{Disclosure Statement}

Die Autoren erklären, dass keine Interessenskonflikte bestehen.

\section{Lizenzangabe}

Machado A, Quadflieg K, Oliveira A, Keytsman C, Marques A, Hansen D, Burtin C: Exercise Training in Patients with Chronic Respiratory Diseases: Are Cardiovascular Comorbidities and Outcomes Taken into Account? - A Systematic Review. J Clin Med. 2019;8(9):1458. (DOI: 10.3390/jcm809145) ( 2019 by the authors. Licensee MDPI, Basel, Switzerland (Übersetzung; Beiträge der einzelnen Autoren, Finanzielle Unterstützung und Danksagungen gekürzt), lizensiert unter CC BY 4.0 (https://creativecommons.org/ licenses/by/4.0/deed.de).

\section{Literatur}

Die Literatur ist unter https://www.karger.com/Article/Fulltext/509305 abrufbar. 